\title{
HCC Immune Surveillance and Antiviral Therapy of Hepatitis C Virus Infection
}

\author{
Solomon Owusu Sekyere ${ }^{a}$ Bernhard Schlevogt ${ }^{\text {a, e }}$ Friederike Mettke ${ }^{a}$ \\ Mohammad Kabbani $^{a} \quad K^{a}$ atja Deterding ${ }^{a}$ Thomas Christian Wirth ${ }^{a}$ \\ Arndt Vogel ${ }^{a} \quad$ Michael Peter Manns ${ }^{a-c}$ Christine Susanne Falk ${ }^{a, d}$ \\ Markus Cornberg ${ }^{a, b}$ Heiner Wedemeyer ${ }^{a-c, f}$ \\ a Department of Gastroenterology, Hepatology and Endocrinology, Hannover Medical \\ School, Hannover, Germany; ${ }^{\mathrm{b}}$ TTU-IICH, German Center for Infection Research (DZIF), \\ Partner Site Hannover-Braunschweig, Hannover-Braunschweig, Germany; ${ }^{\circ}$ Department of \\ General, Abdominal, and Transplant Surgery, Hannover Medical School, Hanover, Germany; \\ dInstitute of Transplantation Immunology (IFB-Tx), Hannover Medical School, Hannover,

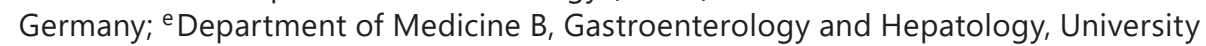

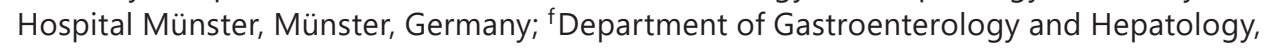 \\ Essen University Hospital, University of Duisburg-Essen, Essen, Germany
}

\section{Keywords}

Hepatocellular carcinoma $\cdot$ Hepatitis C $\cdot$ Immune surveillance $\cdot$ IFN-free therapy $\cdot$ T cells

\begin{abstract}
Objective: HCV clearance by current antiviral therapies improves clinical outcomes but falls short in eliminating the risk for hepatocellular carcinoma (HCC) emergence. As the HCC immune surveillance establishment is vital for the control of neoplastic development and growth, we investigated its correlation with on-/post-treatment HCC emergence, and further analyzed the influence of viral eradication on this setup in patients with HCV-related liver cirrhosis. Design: $\mathrm{PBMC}$ isolated at baseline and longitudinally during therapy were analyzed for tumorassociated antigen (TAA)-specific CD8+ T cell responses against glypican-3 overlapping peptides in vitro using high-definition flow cytometry. Multianalyte profiling of fifty soluble inflammatory mediators (SIM) in the plasma was also performed using Luminex-based multiplex technology. Results: Cirrhosis patients were characterized by an altered profile of distinct SIMs at baseline. At this time point, immune-surveilling T cells targeting specific HCC-associated antigens were readily detectable in HCV-free cirrhosis patients whilst being rather weak in such patients who further developed HCC upon virus eradication. Therapy-induced cure of $\mathrm{HCV}$ infection analogously reduced the strength of the prevailing HCC immune surveillance machinery, particularly by CD8+ T cells in cirrhosis patients. These results were further vali-
\end{abstract}


dated by T cell reactivities to six immuno-dominant HCC-associated HLA-A2-restricted epitopes. Further, we demonstrated that this phenomenon was likely orchestrated by alterations in SIMs - with evidence of IL-12 being a major culprit. Conclusion: Given the relationship between the baseline HCC-specific immune surveilling $T$ cell responses and therapy-associated HCC emergence, and the impact of HCV clearance on its strength and magnitude, we recommend a continued HCC screening in cirrhotic HCV patients despite HCV resolution.

(C) 2018 S. Karger AG, Basel

\section{Introduction}

Hepatocellular carcinoma (HCC) is the second leading cause of cancer deaths worldwide accounting for over 600,000 deaths annually [1]. The majority of all HCC cases occur as a result of chronic liver inflammation and subsequent cirrhosis, which has often been caused by a persistent infection with the hepatitis $\mathrm{C}$ virus (HCV) [2]. A cure for hepatitis $\mathrm{C}$ therefore represents a promising strategy to reduce the future burden of HCC.

Current antiviral therapies for hepatitis $C$ are effective against the virus and may even achieve revolutionary high sustained virologic response (SVR) rates often exceeding 95\%. Despite this remarkable advance, HCV-related cirrhosis and its attendant complications such as HCC still persist in some patients even after achieving SVR as had been predicted before [3]. Current data suggest that achieving SVR, whether with IFN-based or IFN-free antivirals, may only reduce but not completely eliminate the risk of HCC emergence [4-9]. In fact, the absolute risk of HCC at the time of SVR has been demonstrated as being still high, particularly in patients with established cirrhosis $[6,7,9,10]$. As a result, the quest for further understanding and predictability models of how cirrhosis patients receiving antiviral therapy would fare remains a major priority.

Compelling data support the view that the HCC immune surveillance establishment is important for the control of neoplastic development and growth (reviewed in [11]). Support for the role of an immune cancer surveillance system further comes from observations indicating that strong and multispecific anti-tumor $\mathrm{T}$ cell responses early in disease are associated with a better prognosis and patient survival $[12,13]$. Furthermore, immune checkpoint inhibition therapies for many cancers [14] and specifically for HCC [15] are promising new therapeutic approaches underlying the role of immune T cell responses. Indeed, an effective immune surveillance process may prevent nascent cancer development and a reduction of it may lead to the growth and spreading of neoplastic clones of HCC and other malignancies [16, 17]. For HCC arising from underlying cirrhosis in the context of therapy-induced HCV clearance, it is not known whether this is the case. Some recent studies suggest that IFN-free DAA therapy may alter the concentration of soluble inflammatory mediators (SIM) [18] and may evoke immune reconstitution of intrahepatic interferon-stimulated genes [19]. Whether or not such immunological changes influence the prevailing $\mathrm{T}$ cell immune surveillance machinery of HCC is still unclear.

In this study, we used the IFN-free HCV clearance model to investigate the relevance of baseline immune T cell surveillance, its correlates to post-treatment HCC emergence, and whether therapy-induced HCV clearance disturbs this setup in patients with liver cirrhosis. Using T cell responses directed against a panel of glypican-3 (GPC-3) overlapping peptides in vitro as a yardstick, and corroborated by $\mathrm{T}$ cell responses to selected HLA-A2-restricted immuno-dominant HCC epitopes, our results suggest that therapy-induced cure of HCV infection could affect interleukin (IL)-12-mediated HCC surveillance particularly by CD8+ T cells in liver cirrhosis patients. 


\section{Liver Cancer}

\section{Materials and Methods}

Study Population and Ethical Considerations

This study included $49 \mathrm{HCV}$-related liver cirrhosis patients at the liver outpatient clinic of the Hannover Medical School (MHH) who were enrolled to receive IFN-free DAA therapy for hepatitis C. Cirrhosis was mainly diagnosed by liver histology (F5 and F6 according to ISHAK score), transient elastography ( $\geq 14.5$ $\mathrm{kPa}$ ), and definite morphological signs in ultrasound, MRI, and CT as detailed previously [10]. Non-cirrhotic control patients with chronic hepatitis C (CHC) $(n=11)$ and a total of 22 healthy blood donors were also recruited. Overall, the patients with liver cirrhosis at baseline had a median MELD score, albumin, platelets, bilirubin, AST, ALT, and Fibroscan of $9.34 \mathrm{~g} / \mathrm{L}, 96 \mathrm{tsd} / \mu \mathrm{L}, 17 \mu \mathrm{mol} / \mathrm{L}, 90 \mathrm{U} / \mathrm{L}, 87 \mathrm{U} / \mathrm{L}$, and $32 \mathrm{kPa}$, respectively. Surveillance schedule for HCC emergence was routinely performed by ultrasound imaging for all patients in 3-month intervals during antiviral therapy and 6 months after therapy. Of the 49 cirrhosis patients selected, 38 were those who remained free of HCC during the course of IFN-free DAA treatment (herein termed cirrhto-No HCC), while the remaining 11 were those who were HCC-free at baseline but developed tumors during/ after DAA therapy (herein termed cirrh-to-HCC). Although aggressive tumor growth could be identified in single cases, there was no distinct pattern of such overall. Detailed clinical information for individual patients within the cirrh-to-HCC cohort has been provided in online supplementary Table 1 (for all online suppl. material, see www.karger.com/doi/10.1159/000490360). All clinical parameters analyzed here were statistically comparable between the cirrh-to-No HCC and cirrh-to-HCC stratified cohorts. The non-cirrhotic CHC patients had a significantly reduced bilirubin, AST, ALT, and Fibroscan but a higher platelet count compared to the cirrhosis patients. Patient cohorts and characteristics are summarized in Table 1. Categorization of patients into early and advanced cirrhosis groups for specific comparisons was based on Fibroscan and ARFI results. Patients with a Fibroscan of 12.6-25 kPa or ARFI of 1.81-2.8 were classified as early, whilst those with a Fibroscan $>25$ or ARFI $>2.8$ were considered advanced cirrhosis.

Written informed consent was obtained from all patients as part of protocols approved by the local ethics committee of Hannover Medical School. In addition, all investigations were conducted according to the Declaration of Helsinki (1975).

Peptides, HLA Class I Multimers, and Recombinant Proteins

Overlapping peptides spanning the entire length of the GPC-3 protein (18mers overlapping by 10 amino acids) were purchased from ProImmune Ltd. (Oxford, UK). HLA-A*0201-restricted HCC-specific peptides of $\mathrm{AFP}_{137-145}$ (PLFQVPEPV), NY-ESO-1 157-165 (SLLMWITQC), MAGE-A3 ${ }_{112-120}$ (KVAELVHFL), MAGE-A10 $254-262$ (GLYDGMEHL), SSX-2 $41-49$ (KASEKIFYV) and p53149-157 (STPPPGTRV) at purities ranging from 82.78 to $97.99 \%$ were also obtained from ProImmune Ltd. For these HCC-associated epitope-specific peptides, corresponding PE-labeled MHC class I dextramers were obtained from Immudex (Copenhagen, Denmark). Further, HBV-specific 15-mer overlapping peptides covering the core (4 pools from 41 peptides) and polymerase regions (8 pools from 165 peptides) were purchased from ProImmune Ltd. Also, HLA-A*0201-restricted HBV-specific peptides Core $_{18-27}$ (FLPSDFFPSV), Env $183-191$ (FLLTRILTI), Pol $578-581$ (FLLSLGIHL) and Surf $185-194$ (GLSPTVWLSV) at purities of 96.41, 96.43, 98.63, and 97.10\%, respectively, were obtained from ProImmune Ltd. together with their corresponding PE-conjugated dextramers. All peptides were dissolved in sterile endotoxin-free DMSO (Sigma-Aldrich, Munich, Germany), stored at $-80^{\circ} \mathrm{C}$ and used at a final concentration of $10 \mu \mathrm{g} / \mathrm{mL}$ except HBV overlapping peptides which were used at optimum concentrations of $25 \mu \mathrm{g} / \mathrm{mL}$. For in vitro stimulations, premium-grade human IL-12 (Miltenyi Biotec GmbH, Germany) was used at varying concentrations.

\section{Fluorescent Antibodies and Phenotypical Staining}

PBMC were stained with the following mouse anti-human monoclonal antibodies: anti-human IFN $\gamma /$ clone 25723 (R\&D Systems, USA), anti-CD279 (PD1)/clone EH12.2H7, anti-CD38/clone HB-7 and anti-HLA-DR/clone L243 all obtained from BioLegend Inc. (San Diego, CA, USA). All other antibodies such as anti-CD14/clone M5E2, anti-CD19/clone 5J25C1, anti-CD56/clone B159, anti-TNF/clone MAb11, anti-MIP1- $\beta /$ clone D21-1351, anti-CD3/clone SK7, anti-CD4/clone L200, anti-CD4/clone RPA-T4, and anti-CD8/clone SK1 were purchased from BD Pharmingen (Becton Dickinson, Heidelberg, Germany). Dextramer and antibody stainings were performed as described previously [20]. Briefly, for dextramer staining, cryopreserved PBMC samples were thawed and first incubated with the respective dextramers for $20 \mathrm{~min}$ in the dark at room temperature. Cells were then directly stained with appropriate antibodies (prepared as a mastermix) and incubated at room temperature for 10 min in the dark. After 2 washing steps and resuspension in FACS buffer, cells were acquired using an LSR Fortessa flow cytometer (Becton Dickinson, Heidelberg, Germany) and analyzed with FlowJo 
Table 1. Baseline characteristics of study cohorts

\begin{tabular}{|c|c|c|c|c|}
\hline Parameter & Cirrh-to-No HCC & Cirrh-to-HCC & $\mathrm{CHC}$ & Healthy \\
\hline Subjects, $n$ & 38 & 11 & 11 & 12 \\
\hline HLA-A2-positive patients, $n$ & 17 & 5 & 0 & 5 \\
\hline Male sex, $n(\%)$ & $21(55)$ & $8(73)$ & $6(55)$ & $4(33)$ \\
\hline \multicolumn{5}{|l|}{ Age, years } \\
\hline Median & 59 & 60 & 55 & 49 \\
\hline Range & $35-79$ & $47-80$ & $30-74$ & $40-81$ \\
\hline \multicolumn{5}{|l|}{ HCV genotype, $n(\%)$} \\
\hline 1 & $28(74)$ & $9(82)$ & $6(55)$ & n.a. \\
\hline 3 & $10(26)$ & $2(18)$ & $5(45)$ & n.a. \\
\hline \multicolumn{5}{|l|}{ IFN-free therapy, $n$} \\
\hline Sof & 15 & 5 & 3 & n.a. \\
\hline Sof-Dac & 11 & 0 & 2 & n.a. \\
\hline Sof-Sim & 7 & 1 & 0 & n.a. \\
\hline Sof-Velp & 0 & 0 & 1 & n.a. \\
\hline Sof-Led & 3 & 4 & 3 & n.a. \\
\hline Omb-Par-Das & 2 & 1 & 2 & n.a. \\
\hline \multicolumn{5}{|l|}{ Treatment outcome, $n$} \\
\hline SVR & 33 & 11 & 11 & n.a. \\
\hline Relapse & 5 & 0 & 0 & n.a. \\
\hline \multicolumn{5}{|l|}{ Meld score } \\
\hline Median & 9 & 9 & 7 & n.a. \\
\hline Range & $7-18$ & $7-20$ & $6-10$ & n.a. \\
\hline Above median/total, $n(\%)$ & $11 / 38(29)$ & $5 / 11(45)$ & $1 / 11(9)$ & n.a. \\
\hline \multicolumn{5}{|l|}{ Albumin, g/L } \\
\hline Median & 35.5 & 31 & 40 & n.a. \\
\hline Range & $24-43$ & $17-42$ & $37-47$ & n.a. \\
\hline Above median/total, $n(\%)$ & $19 / 38(50)$ & $5 / 11(45)$ & $5 / 11(45)$ & n.a. \\
\hline \multicolumn{5}{|l|}{ Platelets, tsd/ $\mu \mathrm{L}$} \\
\hline Median & 91.5 & 102 & 211 & n.a. \\
\hline Range & $33-346$ & $48-130$ & $113-280$ & n.a. \\
\hline Above median/total, $n(\%)$ & $19 / 38(50)$ & $5 / 11(45)$ & $5 / 11(45)$ & n.a. \\
\hline \multicolumn{5}{|l|}{ Bilirubin, $\mu \mathrm{mol} / \mathrm{l}$} \\
\hline Median & 17.5 & 16 & 10 & n.a. \\
\hline Range & $6-78$ & $9-70$ & $4-21$ & n.a. \\
\hline Above median/total, $n(\%)$ & $19 / 38(50)$ & $5 / 11(45)$ & $4 / 11(36)$ & n.a. \\
\hline \multicolumn{5}{|l|}{ Fibroscan, $\mathrm{kPa}$} \\
\hline Median & 32 & 30.2 & 5.9 & n.a. \\
\hline Range & $14.4-75$ & $17-75$ & $4.1-14.3$ & n.a. \\
\hline Above median/total, $n(\%)$ & $17 / 35(49)$ & $5 / 10(50)$ & $4 / 11(36)$ & n.a. \\
\hline Unknown & $3 / 38(8)$ & $1 / 11(9)$ & n.a. & n.a. \\
\hline \multicolumn{5}{|l|}{ Alanine aminotransferase, U/L } \\
\hline Median & 90.5 & 69 & 74 & n.a. \\
\hline Range & $28-289$ & $21-267$ & $17-394$ & n.a. \\
\hline Above median/total, $n(\%)$ & $19 / 38(50)$ & $4 / 11(36)$ & $5 / 11(45)$ & n.a. \\
\hline \multicolumn{5}{|l|}{ Aspartate aminotransferase, U/L } \\
\hline Median & 86.5 & 106 & 56 & n.a. \\
\hline Range & $39-281$ & $37-182$ & $20-146$ & n.a. \\
\hline Above median/total, $n(\%)$ & $19 / 38(50)$ & $5 / 11(45)$ & $5 / 11(45)$ & n.a. \\
\hline
\end{tabular}

n.a., not applicable; CHC, chronic hepatitis C; Sof, sofosbuvir; Sim, simeprevir; Dac, daclatasvir; Led, ledipasvir; Velp, velpatasvir; Omb, ombitasvir; Par, paritaprevir; Das, dasabuvir. 


\section{Liver Cancer}

\begin{tabular}{l|l|}
\hline Liver Cancer 2019;8:41-65 \\
\hline DOI: 10.1159/000490360 & $\begin{array}{l}\text { (c) 2018 S. Karger AG, Basel } \\
\text { www.karger.com/lic }\end{array}$ \\
\hline
\end{tabular}

Owusu Sekyere et al.: HCC Immune Surveillance and Antiviral Therapy of HCV Infection

Software (TreeStar Inc., San Diego, CA, USA). A multimer-specific CD8+ T cell population upon in vitro culture was considered detectable if the frequency was at least $0.2 \%$ of total CD8+ $T$ cells after deducting the respective frequencies of unstimulated controls. A dump channel (CD14+CD19+CD56+) was used to exclude unspecific events. Respective fluorescence-minus-one control stains were used to enable gating of positive cell populations.

Antigen-Specific Expansion of T Cells in vitro

PBMC were thawed, suspended in AB Medium containing RPMI-1640 supplied with 10\% human AB-Serum (PAN Biotech GmbH, Germany), 1\% non-essential amino acids, 1\% sodium pyruvate, 1\% penicillin/streptomycin, 0.5\% HEPES buffer (GIBCO) and seeded at $3.0 \times 10^{5}$ cells per well in 96-well U-bottomed plates (Sarstedt GmbH, Nümbrecht, Germany). For epitope-specific T cell expansions, cells were stimulated with respective HCC epitope-specific peptides (as indicated), or HBV-specific negative control peptides, at a pre-established optimal concentration of $10 \mu \mathrm{g} / \mathrm{mL}$ and incubated at $37^{\circ} \mathrm{C}$ and $5 \% \mathrm{CO}_{2}$ for 10 days. In all cases, conditions of unstimulated cells were included as negative controls. Cultures were supplemented with $5 \mathrm{IU} / \mathrm{mL}$ human recombinant IL-2 (Invitrogen, Karlsruhe, Germany) on days 3 and 7. Dextramer and intracellular staining were performed on day 10. For proliferation assays using overlapping peptide pools, the carboxyfluorescein diacetate succinimidyl ester (CFSE) staining protocol was employed as described previously [21]. Briefly, PBMC were labeled with $4 \mu \mathrm{M}$ CFSE and stimulated with our 6-pool-organized GPC-3 overlapping peptides or HBV-specific negative control peptides at a final concentration of 10 or $25 \mu \mathrm{g} / \mathrm{mL}$ per peptide for 7 days, respectively. For IL-12 stimulation experiments, CFSE-labeled (for proliferation) or unlabeled (for cytokine secretion) PBMC were stimulated with different concentrations (10, 5, 2.5, and 0.5 $\mathrm{ng} / \mathrm{mL}$ ) of IL-12 in the presence or absence of GPC-3 overlapping peptide pools. Conditions of DMSO-stimulated cells were used as negative controls as were PHA as positive controls. DMSO concentration did not exceed $0.2 \%$ in all cultures. For the CFSE assay, supplementation of cultures with $5 \mathrm{IU} / \mathrm{mL}$ human recombinant IL-2 was done on day 4.

\section{Intracellular Cytokine Detection}

Intracellular cytokine staining was performed to measure the levels of IFN $\gamma$, MIP1- $\beta$, and TNF by flow cytometry after 10 days of in vitro culture. Cells were resuspended in fresh $\mathrm{AB}$ medium and then restimulated with appropriate peptide concentrations. For IL-12 stimulation assays, appropriate stimulating concentrations of IL-12 were further used. Cells were incubated for a total of $6 \mathrm{~h}$ with the addition of $2 \mu \mathrm{g} / \mathrm{mL}$ Brefeldin A (Sigma-Aldrich, Germany) 1 h into the culture period. Cells stimulated with phorbol 12-myristate 13-acetate (PMA) and Ionomycin were used as positive controls. Surface staining for CD3, CD4, CD8 as well as CD14/19/56 was performed before intracellularly staining for the cytokines IFN $\gamma$, MIP1- $\beta$, and TNF as detailed before [20]. Cytokine-producing cells in the stimulated condition of frequencies of at least $0.3 \%$, after deducting respective frequencies of unstimulated controls, were considered positive.

\section{Measurement of Plasma Concentrations of SIM}

Multianalyte profiling of plasma concentrations of 50 SIM (SIMs) was comprehensively performed in a single run using the LUMINEX-based multiplex technology (BioPlex Pro Human Cytokine Panel; Bio-Rad, Hercules, CA, USA) according to the manufacturer's guidelines as detailed previously [22] and as per optimized protocols used in various previous studies [21, 22].

\section{Statistical Analyses}

Data analysis was performed using GraphPad Prism software (GraphPad Software, La Jolla, CA, USA). For descriptive purposes, statistics were expressed as mean values \pm standard error of mean. Generally, $p$ values of $<0.05$ were considered to be statistically significant * $^{*}<<0.05$; ${ }^{* *} p<0.01$; *** $p<0.001$; ${ }^{* * * *} p<$ $0.0001)$. Detailed information on statistical tests used for each figure is outlined in the respective figure legends.

\section{Results}

Serum Concentrations of Various SIM Are Altered in Patients with HCV-Related Liver Cirrhosis

An inflammatory microenvironment is considered to be an essential component of all tumors and plays a critical role in tumorigenesis. Pursuant to our earlier report of altered SIM 


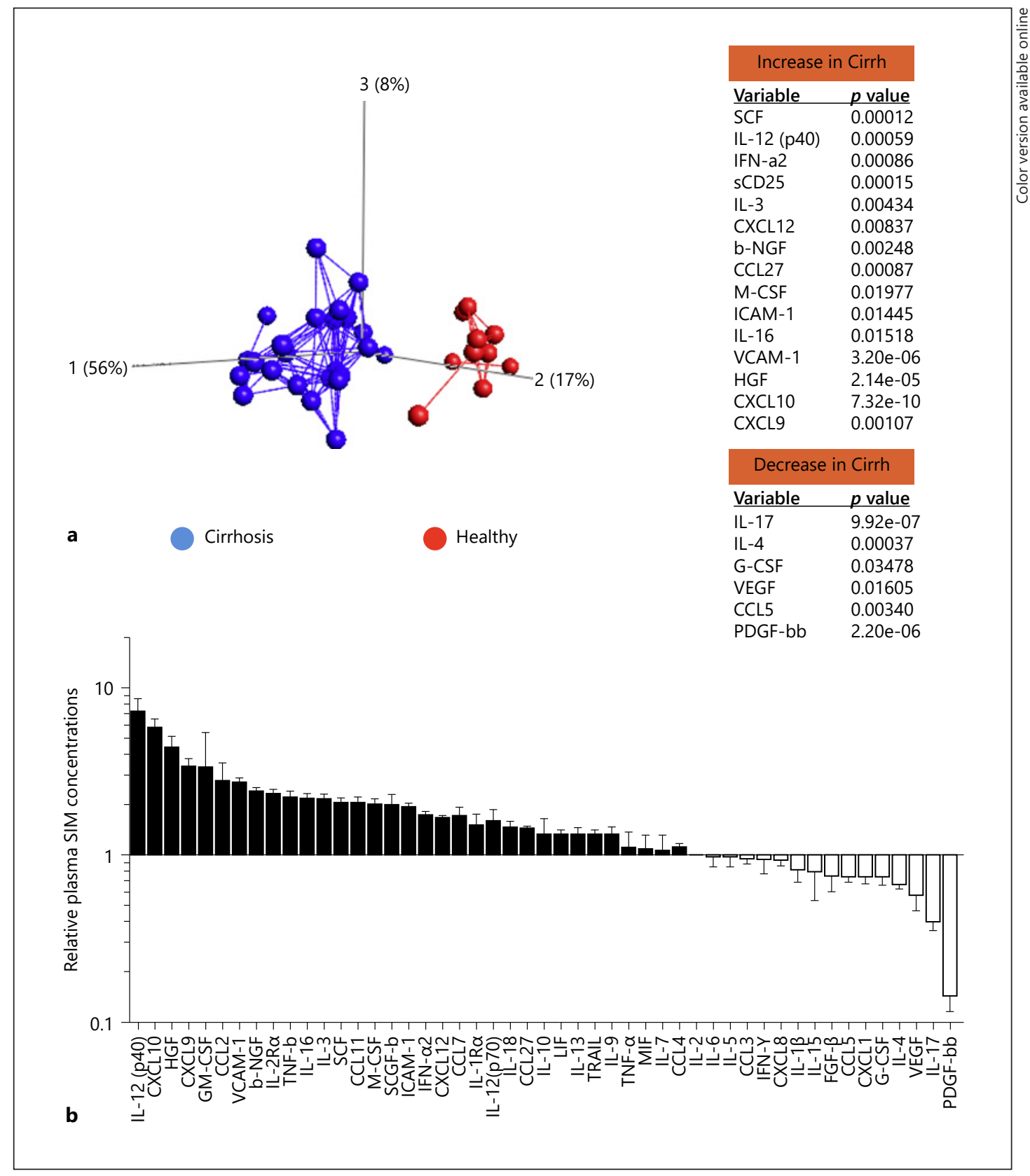

Fig. 1. Differential concentrations of soluble inflammatory mediators (SIMs) in patients with liver cirrhosis relative to healthy donors. The concentrations of 50 SIMs were measured in the plasma from cirrhosis patients without any detectable tumors prior to antiviral therapy $(n=24)$ as well as from healthy individuals $(n=10)$ using the multiplex assay. a Principal component analysis plot showing the distinct clustering at baseline based on the differential levels of the SIMs measured. SIMs that significantly increased (top panel) or decreased (lower panel) are accordingly indicated. b The relative SIM profiles of the cirrhosis patients with respect to normal levels in healthy individuals. Principal component analysis was performed using Qlucore Omics explorer Software v2.2 (Qlucore AB, Lund, Sweden). A $t$ test was used for the 2-group comparison. 


\section{Liver

concentrations observed in a disparate cohort of CHC patients (i.e., with/without cirrhosis) [18], we first aimed to evaluate the extent to which the inflammatory milieu is altered in a severely sick cohort consisting entirely of patients with advanced cirrhosis with/without post-treatment HCC. Using the LUMINEX-based multiplex assay technology, we assessed the serum profiles of 50 SIM at baseline between cirrhosis patients $(n=24)$ and healthy controls ( $n=10$ ) (Fig. 1). By Principal Component Analysis, we observed that patients with cirrhosis differed substantially from healthy controls. This was evidenced by a clearly distinct clusterization of either cohort (Fig. 1a). Further evaluating the relative concentrations of individual SIMs in the cirrhosis patients, we could observe a more heightened inflammation in this cohort compared to healthy controls. Notably in the cirrhotic cohort, IL-12(p40) and IL-12(p70) were not just significantly increased relative to healthy controls but the former (i.e., IL-12p40), in particular, was the most significantly upregulated SIM (Fig. 1b). Overall, these data thus revealed a profoundly altered inflammatory profile at baseline in cirrhosis patients.

\section{GPC-3-Specific T Cell Responses Are Readily Detectable in Patients with Liver Cirrhosis}

Relative to Non-Cirrhotic Controls

In order to study the possible effect the altered inflammatory milieu may have on baseline HCC immune surveillance, we investigated the prevalence, magnitude, and breadth of circulating GPC-3-specific T cells in patients with liver cirrhosis without any detectable tumors upon achieving SVR with IFN-free therapy. To aid our quest, we established an in vitro HCCassociated antigen stimulation protocol using overlapping peptides spanning the entire length of GPC-3, organized into 6 pools (see online suppl. Table 2). Using this assay, cytokineproducing GPC-3-specific CD4+ and CD8+ T cells were detected by intracellular cytokine secretion upon a 10-day stimulation of PBMC isolated before antiviral therapy from cirrh-toNo HCC patients, or from non-cirrhotic controls (Fig. 2a). Our results showed significantly stronger and broadly directed IFN $\gamma$-producing CD4+ and CD8+ T cells detectable in the cirrhotic patients upon stimulation with GPC-3-specific peptides (Fig. 2b) but not with negative control peptides in selected HBsAg-negative, anti-HBsAg/anti-HBcAg-negative patients (see online suppl. Fig. 1A, 1B). While the breadth (mean number of recognized pools/ patient) of IFN $\gamma$-secreting GPC-3-specific T cell responses in cirrhotic patients displayed a clear trend of superiority compared with $\mathrm{CHC}$ patients and healthy individuals, the effective strength (overall mean frequency/patient) was overwhelmingly higher as compared to both groups of non-cirrhotic subjects (Table 2a).

For MIP1- $\beta$ and TNF secretion, there was no significantly substantial alterations observable between the 3 study cohorts (see online suppl. Fig. 2). Using Boolean analysis to explore the baseline response status of polyfunctional GPC-3-specific T cells, however, we observed that both CD4+ and CD8+ T cells expanded with GPC-3 peptides in vitro exhibited a pleiotropic functionality in the cirrhotic cohort with significantly higher polyfunctional (IFN $\gamma+\mathrm{MIP} 1-\beta+\mathrm{TNF}+$ ) tendencies relative to non-cirrhotic controls (Fig. 2c).

We next sought to investigate whether stimulation with HCC-associated antigens was also able to increase $\mathrm{T}$ cell proliferative capacity in the cirrhotic cohort. Here, both the breadth and magnitude of GPC-3-specific CD4+ and CD8+ T cell proliferative responses were much higher in the cirrhotic patients as compared to the non-cirrhotic controls (Fig. 2d, e) (Table $2 b$ ). In a parallel stimulation experiment with PHA, it was further observed that PBMC from the non-cirrhotic controls did not harbor any inherent trait of proliferative impairment (Fig. 2f). Furthermore, stimulation of PBMC from selected HBsAg-negative, anti-HBsAg/anti$\mathrm{HBcAg}$ cirrhosis patients with HBV-specific negative control antigens did not amount to a similar observable proliferative capacity, further affirming the specificity of the GPC-3-specific $\mathrm{T}$ cell responses (see online suppl. Fig. 1C). Of note, comparing baseline responses did 


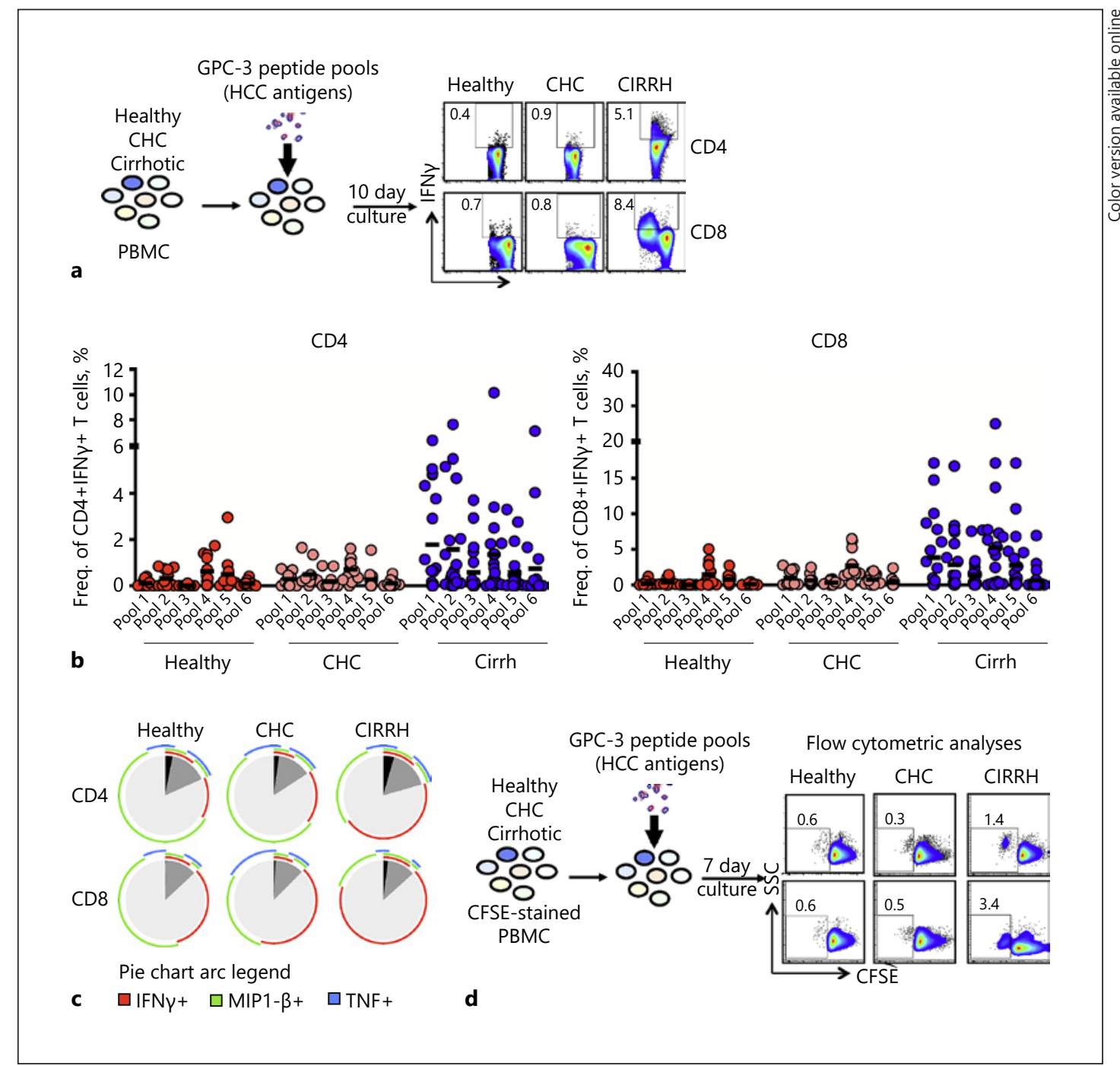

Fig. 2. The magnitude and breadth of TAA-specific T cell responses in cirrhosis patients before antiviral therapy for HCV. Detectability of circulating TAA-specific T cell responses to overlapping peptides covering the entire length of GPC-3 were tested in cirrhosis patients and non-cirrhotic controls at baseline in vitro. a Representative FACS plots of IFN $\gamma$ production compared between cirrhosis patients, non-cirrhotic CHC patients, and healthy individuals are shown. b IFN $\gamma$-producing GPC-3-specific CD4+ and CD8+ T cells measured by intracellular cytokine staining upon a 10-day stimulation with HCC-associated peptides. c Boolean analyzed polyfunctional (IFN $\gamma+\mathrm{TNF}+\mathrm{MIP} 1-\beta+$ ) capabilities of GPC-3-specific CD4+ and CD8+ T cells between cirrhosis patients and the non-cirrhotic controls. Mean values of all subjects in each cohort are represented. d Representative FACS plots showing proliferative responses by GPC-3-specific T cells in the three cohorts as measured by a CFSE proliferation assay in a 7-day culture in vitro. e Proliferation of GPC-3-specific CD4+ and CD8+ T cells in the cirrhosis cohort relative to non-cirrhotic controls. $\mathbf{f}$ Proliferative responses of CD4+ and CD8+ T cells to PHA stimulation in cirrhosis patients compared to those by non-cirrhotic controls. Red horizontal bars indicate mean values. All responses for stimulated cultures as plotted were those obtained after deducting background frequencies. Ordinary one-way ANOVA was the statistical test employed. Cirrh, cirrhosis; CHC, chronic hepatitis C; /IFN $\gamma$, interferon gamma; MIP1- $\beta$, macrophage inflammatory protein 1- $\beta$; $\mathrm{TNF}$, tumor necrosis factor.

(Figure continued on next page.) 


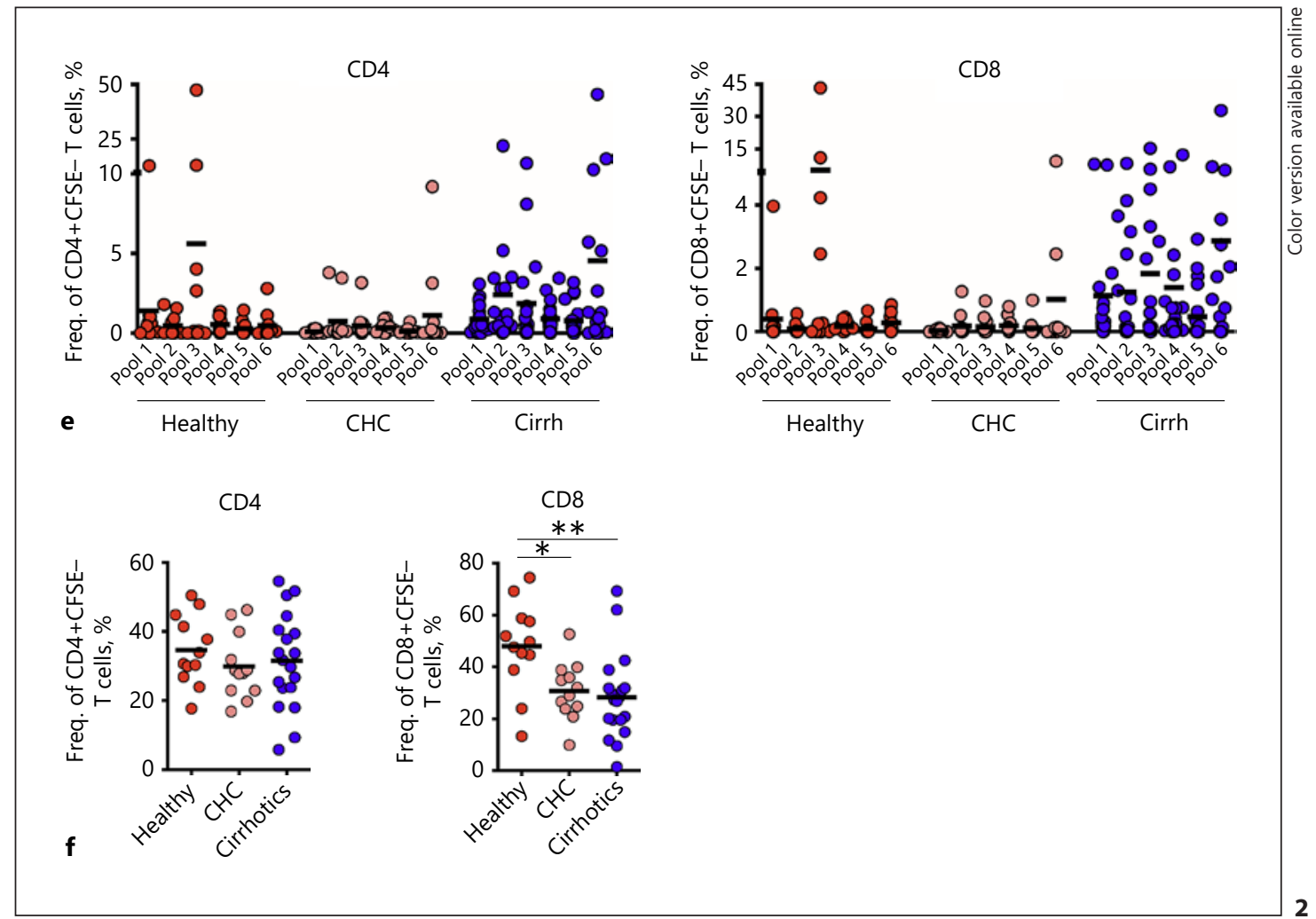

not reveal any significant differences between early and advanced cirrhosis patients, although a clear trend of elevated IFN $\gamma$ secretion was observed in the latter (see online suppl. Fig. 3A, B).

GPC-3-Specific T Cell Responses Are Rarely Detectable prior to Antiviral Therapy in

Patients Who Develop Post-Treatment HCC

The robust GPC-3-specific $\mathrm{T}$ cell responses prior to antiviral therapy that prevailed in the cirrh-to-No HCC cohort prompted us to further evaluate the relevance of a stronger baseline immune-surveilling T cell response in inhibiting tumor cell proliferation upon HCV antiviral treatment. To do this, we compared the pre-antiviral-therapy CD4+ and CD8+ T cell cytokine production and proliferation in patients with baseline cirrhosis who developed post-IFN-free DAA treatment and those that did not (Fig. 3a). The cirrh-to-HCC patients displayed a sharply reduced cytokine secretion potential compared to the cirrh-to-No HCC patients (Fig. 3b-d).

Similarly for proliferation, we observed a significantly reduced GPC-3-specific CD4+ and CD8+ T cell capacity in the cirr-to-HCC compared to cirrh-to-No HCC patients (Fig. 3e). Notably, the low baseline cytokine (IFN $\gamma$, MIP1- $\beta$, TNF) secretion as well as proliferation was observed across both cohorts of de novo and recurrent HCC patients (data not shown). These results highlighted the particular relevance of a potent baseline immuno-surveillance system in warding off the proliferation of neoplastic clones that may develop upon antiviral treatment of hepatitis C.

DAA Therapy for HCV Significantly Decreases the Strength of GPC-3-Specific CD8+ but Not CD4+ T Cell Responses in Cirrhotic Patients

Our previous study in patients with $\mathrm{CHC}$ receiving IFN-free DAA therapy revealed profound changes in SIM concentrations predominantly characterized by a downregulated 
Table 2. Summary of the frequency, breadth, and magnitude of IFN $\gamma$-secreting and proliferative HCC-specific T cell responses in the different study cohorts

$\begin{array}{lll}\text { Healthy } & \text { CHC } & \text { Cirrh } \\ (n=12) & (n=11) & (n=21)\end{array}$

a Summary of responses by IFN $\gamma$-secreting T cells in cirrhotic patients and non-cirrhotic controls

\begin{tabular}{|c|c|c|c|c|}
\hline Patients responding to at least one pool, $\%$ & $\mathrm{CD} 4$ & 58 & 82 & 62 \\
\hline & CD8 & 67 & 100 & 81 \\
\hline Patients responding to more than 3 pools, $\%$ & CD4 & 0 & 9 & $29^{\dagger}$ \\
\hline & CD8 & 17 & 27 & 38 \\
\hline Mean number of recognized pool/patient & $\mathrm{CD} 4$ & 1.00 & 1.73 & 2.24 \\
\hline & CD8 & 1.58 & 2.91 & 2.67 \\
\hline Mean frequency of recognized pool/patient, \% & $\mathrm{CD} 4$ & 1.17 & 1.59 & $6.02 *, \dagger$ \\
\hline & CD8 & 2.57 & 5.70 & $15.71^{*, \dagger}$ \\
\hline Overall mean frequency per patient, $\%$ & CD4 & 1.64 & 2.09 & $6.34^{*, \dagger}$ \\
\hline & CD8 & 2.85 & 6.07 & $15.94^{*, \dagger}$ \\
\hline b Summary of proliferative $T$ cells responses in & ents a & hotic ce & & \\
\hline Patients responding to at least one pool, \% & $\mathrm{CD} 4$ & 92 & 75 & 95 \\
\hline & CD8 & 83 & 67 & $95^{*}$ \\
\hline Patients responding to more than 3 pools, $\%$ & $\mathrm{CD} 4$ & 17 & 25 & $55^{\dagger}$ \\
\hline & CD8 & 0 & 0 & $35^{*, \dagger}$ \\
\hline Mean number of recognized pool/patient & $\mathrm{CD} 4$ & 2.33 & 2.09 & $3.76^{*, \dagger}$ \\
\hline & CD8 & 1.67 & 1.00 & $2.57^{*}$ \\
\hline Mean frequency of recognized pool/patient, $\%$ & $\mathrm{CD} 4$ & 8.54 & 2.85 & $10.63^{*}$ \\
\hline & CD8 & 5.99 & 1.63 & $8.39 *$ \\
\hline Overall mean frequency per patient, $\%$ & $\mathrm{CD} 4$ & 8.78 & 3.13 & $10.76^{*}$ \\
\hline & CD8 & 6.17 & 1.80 & $8.53^{*}$ \\
\hline
\end{tabular}

CHC, chronic hepatitis C; cirrh, cirrhosis. ${ }^{*} p<0.05$ vs. CHC; ${ }^{\dagger} p<0.05$ vs. healthy. The frequency of CD4+/CD8+IFN $\gamma+$ T cells was considered positive if the result upon deducting the response from unstimulated controls was $\geq 0.5 \%$. An independent group's $t$ test was performed to compare the cirrhosis patients with CHC or healthy donors. The $t$ statistic was considered significant at a 0.05 significance level.

outlook [18]. To better understand whether such/suchlike immunological changes following cure of HCV alter the prevailing HCC immune surveillance machinery in cirrh-to-No HCC patients, we longitudinally assessed and compared cytokine production and proliferation by GPC-3-specific T cells at therapy start (TS), end of therapy (EOT) and upon follow-up (FU) in vitro. Overall, the secretion of cytokines such as IFN $\gamma$, MIP1- $\beta$, and TNF by GPC-3-specific CD8+ T cells experienced significant reductions at EOT and/or FU (Fig. 4a, b). At individual patient level, however, the reduction in strength of cytokine-producing GPC-3-specific CD8+ $\mathrm{T}$ cells was not a unidirectional phenomenon. As a result, we calculated and compared the respective percentage changes in responses at EOT and FU relative to baseline values. For all three individual cytokines, the number of individual responses that significantly decreased generally overweighed those that either remained unchanged or increased (Fig. 4c). Probing the fate of polyfunctional GPC-3-specific CD8+ T cells, we further observed that the simultaneous secretion of two/three cytokines was also more significantly reduced at an even more pronounced degree (Fig. 4d). Apart from IFN $\gamma$ production that declined at FU, cytokine production by GPC-3-specific CD 4+ T cells generally did not show any such declines. A similar observation was also made for GPC-3-specific CD4+ T cells' polyfunctionality. Further investigating the dynamics of $\mathrm{T}$ cell responses in more homogeneous cirrhosis groups according to the stage of liver disease, we observed that while the reduction in the functional capacity of GPC-3-specific CD4+ T cells to secrete IFN $\gamma$ depended on the cumulative declines in both 


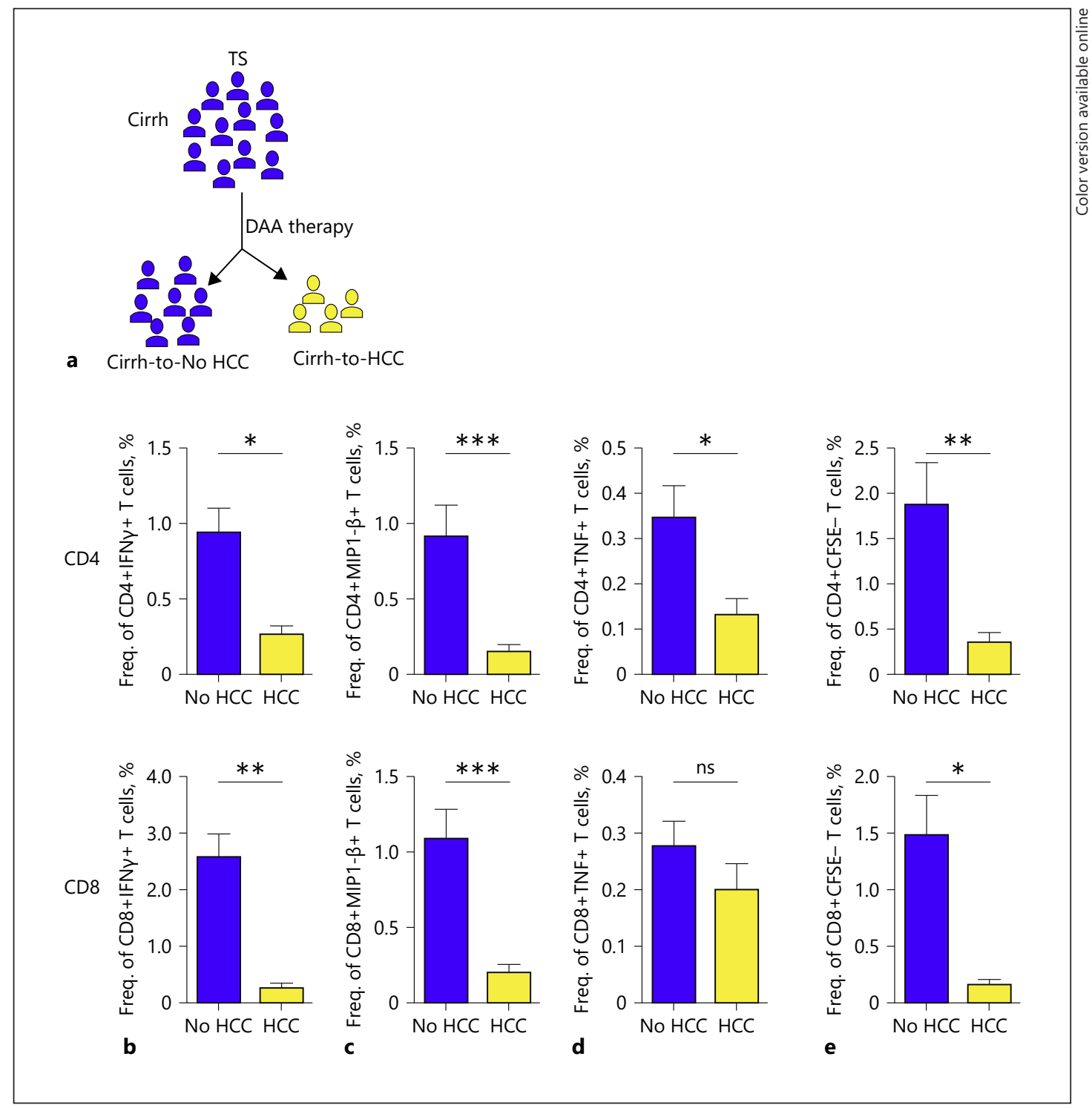

Fig. 3. The strength of TAA-specific T cell responses at baseline in cirrhosis patients that developed HCC following DAA therapy. a Schematic representation of the parent cirrhotic cohort and delineated subgroups of "cirrh-to No HCC" and "cirrh-to-HCC" whose T cell responses to GPC-3-specific peptides were specifically compared at baseline. Secretion of IFN $\gamma$ (b), MIP1- $\beta$ (c), and TNF (d) by TAA-specific CD4+ and CD8+ T cells upon 10-day culture in the presence of GPC-3 overlapping peptides. e Proliferative responses of GPC-3-specific CD4+ and CD8+ T cells measured by a CFSE proliferation assay after 7-day culture. Background responses from unstimulated controls were deducted in all cases. The Mann-Whitney test was used to statistically compare groups. TS, therapy start; Cirrh, cirrhosis; IFN $\gamma$, interferon gamma; MIP1- $\beta$, macrophage inflammatory protein 1- $\beta$; TNF, tumor necrosis factor.

early and advanced cirrhosis groups, that by GPC-3-specific CD8+ T cells was significantly reflected in patients with advanced rather than early cirrhosis (see online suppl. Fig. 3C).

Similar to cytokine production, the resultant magnitude of GPC-3-specific CD8+ T cell proliferation, but not that for CD4+s, showed significant decrements at EOT and FU (Fig. 4e). In accordance with the effective decline of the GPC-3-specific CD8+ T cell proliferative responses, we observed that a significantly higher number of individual responses were 
Liver

Liver Cancer 2019;8:41-65

52
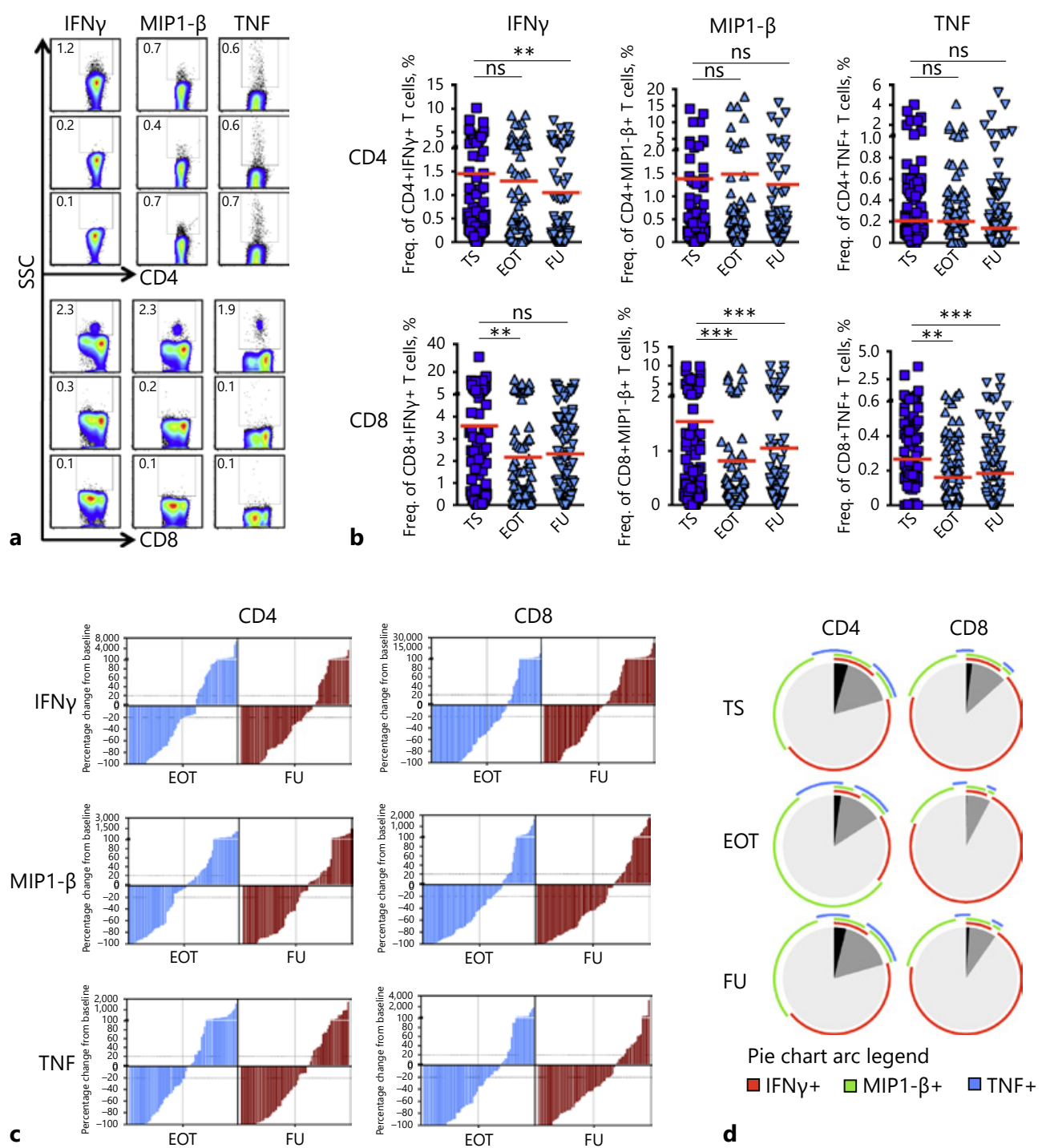

Pie chart arc legend $\square I F N{ }^{+} \quad \square$ IP- $\beta+\quad \square T N F+$

CD38

Pool 1 Pool 2 Pool 3 Pool 4 Pool 5 Pool 6 DMSO

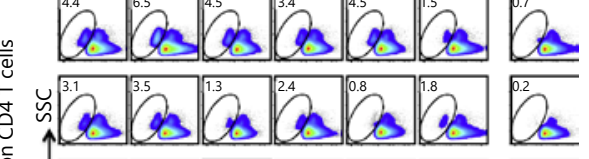
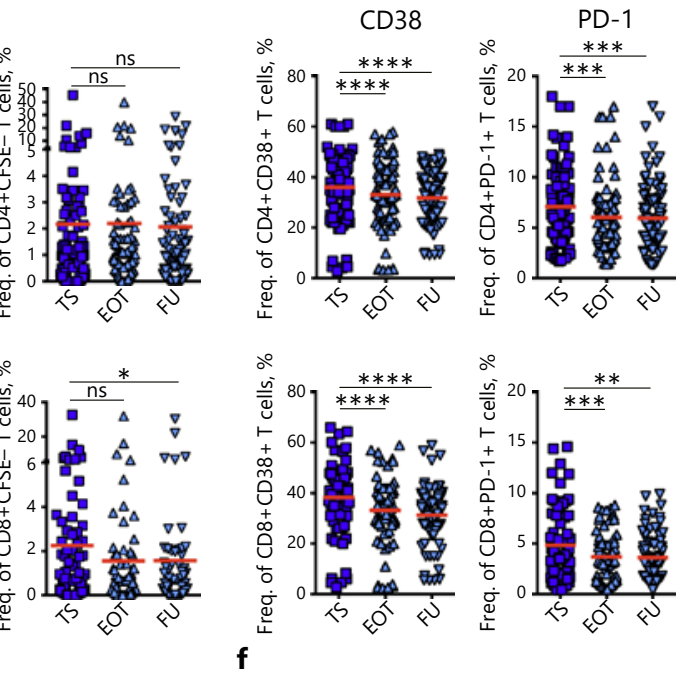

LARGER

(For legend see next page.) 


\section{Liver

particularly reduced at FU compared to those that either remained unchanged or even increased (see online suppl. Fig. 4A). Expectedly, the distribution of "increased," "decreased," and "unchanged" proliferative responses was not significantly altered for GPC-3-specific CD4+ T cells (see online suppl. Fig. 4A). The breadth of proliferative responses for both GPC3 -specific $\mathrm{T}$ cell populations was however not significantly altered (data not shown). Also, the proliferation of both GPC-3-specific CD4+ and CD8+ T cells was not significantly different between early and advanced cirrhosis groups (see online suppl. Fig. 3D).

To extend our observations to other T cell attributes, we further assessed the expression of important in vitro T cell activation markers CD38 and PD-1 on GPC-3-specific CD8+ and CD4+ T cells in responding cultures. Significantly, the expression of both CD38 and PD-1 that were elevated in stimulated cultures consistently declined from TS through EOT to FU (see online suppl. Fig. 4B) (Fig. 4f).

GPC-3-Specific T Cell Responses Remain Weak during IFN-Free Therapy in Patients That

Developed On-/Post-Treatment HCC, but Suddenly Appear to Coincide with HCC

Emergence

We next sought to analyze the CTL responses to GPC-3-specific peptides in the cirrh-toHCC patients. PBMC samples were available for $8 / 11$ patients at the designated time points of TS, EOT, and FU for such studies. As the cumulative analysis of data generated from a patient cohort like this one consisting of patients with varying clinical HCC emergence and progression profiles has a risk of clouting the representative picture in its truest sense, we resorted to analyzing each patient individually. There was no distinctive $\mathrm{T}$ cell response pattern between de novo HCC $(n=5)$ and recurrent HCC patients $(n=3)$. In both cohorts alike, and as expected, IFN $\gamma$ secretion by GPC-3-specific CTLs strongly increased coincidentally with the emergence of HCC - after they had been very weak or absent before HCCs diagnosis. We further subclustered the patients into "early" and "late" HCC developers according to the evolution of tumor after the initiation of antiviral therapy. HCCs identified within 4 months after TS were described as "early HCC," while those that emerged beyond this time point were put in the "late HCC" bracket (Fig. 5a). Using this categorization, we observed that the strong IFN $\gamma$-secreting capacity by GPC-3-specific CTLs detected in coincidence with HCC emergence in the early HCC patients did not further increase but rather either remained fairly unchanged (i.e., in 2/5 patients: patients 3 and 4 ) or declined in breadth and/or magnitude in the remaining $3 / 5$ patients (patients 1,2 , and 5 ) after the tumors had been successfully treated (Fig. 5b, c). The dynamics of CTL responses in the patients who developed early HCC, however, could not be very well examined between TS and EOT (where strong T cell responses were observed) because PBMCs were not sampled between these two time points. Further analyzing GPC-3-specific CTL responses in the late HCC subcohort $(n=3)$, it became apparent that the weak CTL responses initially present before antiviral therapy either declined, or remained

Fig. 4. The fate of TAA-specific T cell responses in cirrhosis patients following antiviral therapy for hepatitis C. HCC-associated T cell responses to GPC-3 overlapping peptides were longitudinally assessed in cirrhosis patients at therapy start (TS), end of therapy (EOT) and at 12 or 24 weeks' follow-up (FU) in vitro. a Representative FACs plots for cytokine secretion. Secretion of individual (IFN $\gamma$, MIP1- $\beta$, TNF) (b) as well as two or more cytokines (c) by GPC-3-specific CD4+ and CD8+ T cells compared between the three time points. d Percentage change of cytokine-secreting responses to individual pools by GPC-3-specific CD4+ and CD8+ T cells at EOT and FU from respective baseline responses. e Representative FACS plots (left) and pooled data (right) of proliferative responses by GPC-3-specific CD4+ and CD8+ T cells at TS, EOT, and FU. $\mathbf{f}$ Expression of CD38 and PD-1 by GPC-3-specific CD4+ and CD8+ T cells upon 7 days of stimulation with GPC-3 overlapping peptides. Statistical analysis performed with two-tailed Wilcoxon matched-pairs signed rank test. Horizontal bars represent means. PD-1, programmed cell death protein 1. 




Fig. 5. Longitudinal dynamics of CTL responses to GPC-3-specific peptides in cirrhosis patients who developed HCC following IFN-free therapy. GPC-3-specific T cell responses were assessed at therapy start (TS), end of therapy (EOT), and follow-up (FU) in the subgroup of cirrhosis patients who developed on-/posttreatment HCC upon receiving IFN-free DAA therapy for hepatitis C. a Categorization of patients into "early" and "late" HCC developers according to the time of HCC emergence after initiation of antiviral therapy. Representative FACS plots of 1 early (b) and 1 late HCC patient (d) indicating the changes in frequency of IFN $\gamma$ secreting CD8+ T cells during the course of DAA therapy and beyond. Bar graphs showing longitudinal IFN $\gamma$ secretion by GPC-3-specific CD8+ T cells for individual patients within the early (c) and late (e) HCC cohorts are displayed. Note: for Pt. \#2, the earliest time point sample available was TW6, which was used instead of one from TS.

(Figure continued on next pages.)

fairly so at best, until the time of initial HCC diagnosis, where strong T cell responses coemerged (Fig. 5d, e). These GPC-3-specific IFN $\gamma$ responses for CD8+ T cells were also similar to those observed for CD4+ T cells as shown for "early HCC" (see online suppl. Fig. 5A, B) and "late HCC" (see online suppl. Fig. 5C, D).

We further performed comparative analysis between the two cirrh-to-HCC and cirrh-toNo HCC groups. It was clear that at any time point (be it TS, EOT, or FU), the magnitude of IFN $\gamma$-secreting GPC-3-specific CD4+ and CD8+ T cell responses in the cirrh-to-No HCC patients was significantly higher than the cirrh-to-HCC (see online suppl. Fig. 6A, B, and online suppl. Table 3). Interestingly, even at FU where those responses were significantly increased in the 

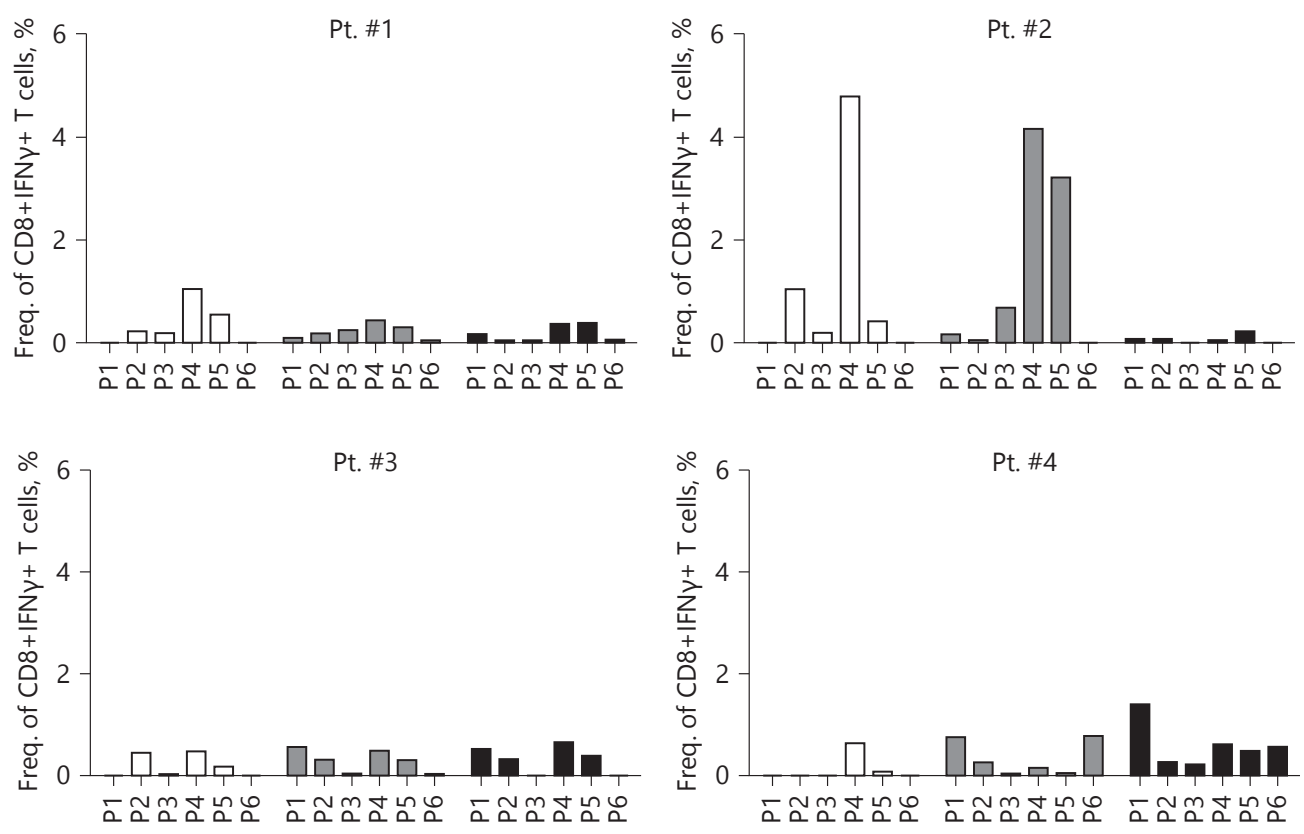

c
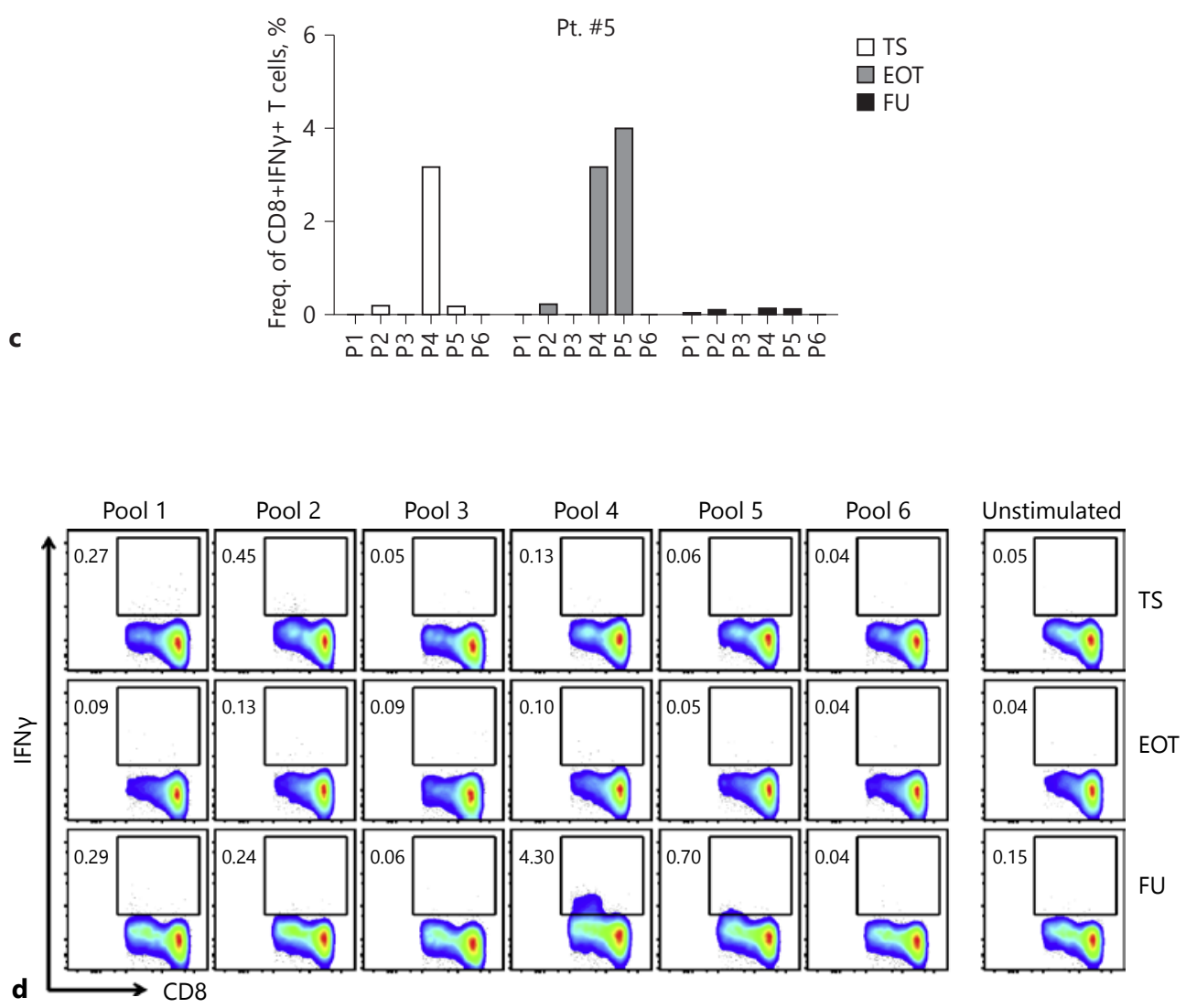

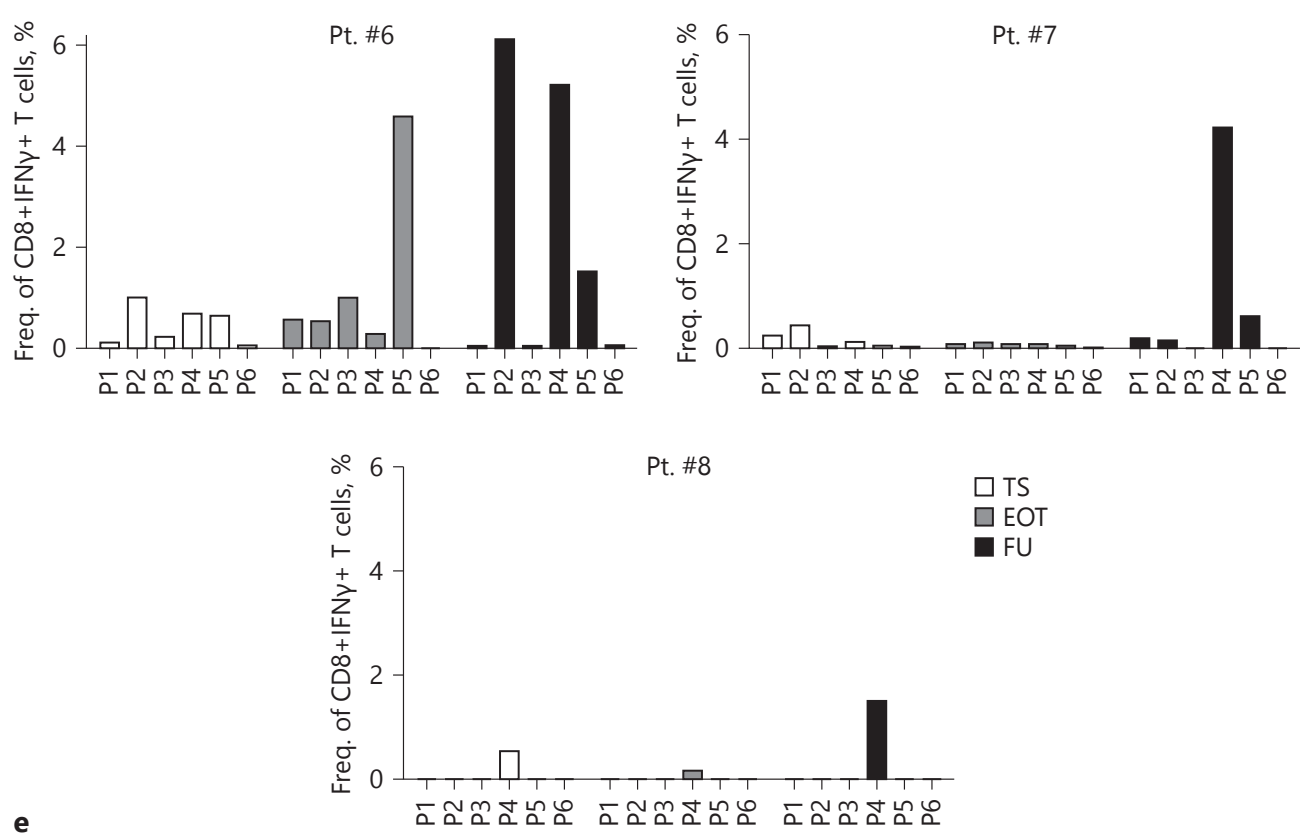

5

late HCC subgroup of the cirrh-to-HCC patients, the strength of response was still significantly lower by statistical comparison to cirrh-to-No HCC (see online suppl. Table 3).

The Strength of HCC Epitope-Specific CD8+ T Cell Responses Decreases upon DAA Therapy for Hepatitis $C$

Epitope-specific CD8+ T cells are present in the natural T-cell repertoire and play a central role in the pathogenesis of HCC. As a next step, we aimed to seek evidence for a possible effect of antiviral therapy for hepatitis $C$ on this $T$ cell population. In particular, we focused on responses directed against the highly immunogenic HLA-A2-restricted epitopes of AFP137-145, NY-ESO-1157-165, MAGE-A3112-120, MAGE-A10254-262, SSX-241-49 and p53149-157 that are well characterized in HCC [23]. PBMC from cirrh-to-No HCC patients ( $n=17$ ) isolated at TS, EOT, and FU were stimulated with the specific peptides in vitro. HBVspecific Core ${ }_{18-27}$, Env $183-191$, Pol $_{578-581}$, and Surf ${ }_{185-194}$ control peptide stimulations were used in selected patients with no HBV coinfection to secure the quality of staining (see online suppl. Fig 7). The secretion of MIP1- $\beta$ at some time point to one of the six epitopes, at least, was detected in most of the patients. In most cases, these responses were those directed against NY-ESO-1 and MAGE-A10 and the others to a lesser extent. Overall, where significant MIP1- $\beta$-producing CD8+ T cell responses were detected, they were essentially robust at TS but waned at EOT and/or FU (Fig. 6a-c). IFN $\gamma$ and TNF secretions were rather generally weak as significant levels were only detected at isolated instances. No cytokine-producing CD8+ T cell responses were detected upon stimulation with irrelevant control peptides (see online suppl. Fig 7A, B).

The proliferation of TAA-specific CD8+ T cells was further evaluated by staining with respective MHC class I dextramers. Identification of tumor-specific CD8+ T cell responses by this technique was a relatively rare event. In all, significant proliferative responses at some time point to at least one epitope were observed in $6 / 17$ patients upon stimulation with 


\section{Liver Cancer}

respective TAA-specific peptides but not with negative control peptides (see online suppl. Fig 7C, D). The number of significant responses totaled 11 and was mostly directed against NY-ESO-1 and MAGEA3/A10. Again with this readout, we observed substantial general reductions of the strong baseline proliferative capacities of TAA-specific CD8+ T cells following antiviral therapy for hepatitis C (Fig. $6 \mathrm{~d}$ ). In the overall data, a statistically significant abrogation of baseline multimer-specific CD8+ T cell proliferation at EOT and FU became more evident (Fig. 6e).

In vitro Stimulation with IL-12 Endowed GPC-3-Specific T Cells with Enhanced Activity

The pathogenesis of HCC due to chronic liver disease involves stimulation of the immune system which may not only cause persistent inflammation and fibrosis but also a possible enhancement of immune-surveilling $\mathrm{T}$ cell responses in a bid to check the proliferation of cancer cells. To provide experimental evidence that SIM may indeed contribute to the strong baseline presence of GPC-3-specific T cell responses in cirrhotic patients, we narrowed in on IL-12 and examined its role in this frame in vitro. PBMC from cirrhotic patients isolated before antiviral treatment were stimulated with different concentrations of IL-12 in the presence (or absence) of GPC-3 overlapping peptide pools. Indeed, stimulation with higher concentrations of IL-12 resulted in enhanced secretion of the cytokines IFN $\gamma$, MIP1- $\beta$, and TNF by both GPC3 -specific CD4+ and CD8+ T cells in a similar fashion compared with stimulation with peptide alone. Importantly, the intensity of cytokine production observed with higher concentration of IL-12 stimulation reduced sequentially to stimulation with reducing concentrations (Fig. 7a-d).

Analyzing the proliferative capacities, however, we observed that stimulation with higher concentrations of IL-12 resulted in a significantly robust expansion only of GPC-3-specific CD8+ but not CD4+ T cells, reminiscent of our earlier observation. Here too, for the GPC3-specific CD8+ T cell responses, decreasing concentrations of IL-12 led to a consistent dosedependent lessening of the magnitude (Fig. 7e, f). Interestingly, we could further show that although the longitudinally measured plasma levels of IL-12 for both cirrh-to-No HCC and cirrh-to-HCC patients significantly reduced following HCV resolution, their respective levels at the specific time points were differentially higher in cirrh-to-No HCC patients (Fig. $7 \mathrm{~g}$ ) in accordance with a stronger $\mathrm{T}$ cell response profile.

For both cytokine secretion and proliferation, the effect of IL-12 was not restricted to particular pools as all pools responded alike (data not shown). Also of note, the stimulation of baseline PBMC from cirrhotic patients with IL-12 alone without any GPC-3-specific peptides did not result in any significant alterations of the functionality and proliferative capacity of both CD8+ and CD4+ GPC-3-specific T cells, suggesting a predominant antigen-dependent effect of IL-12 in modulating these T cell responses (data not shown).

\section{Discussion}

In this study, we comprehensively probed the HCC immune surveillance system existent in patients with liver cirrhosis before antiviral therapy and evaluated its relation to on-/posttreatment HCC emergence, using the IFN-free HCV treatment model. Specifically, we characterized the frequency, magnitude and breadth of immuno-surveilling HCC-specific T cells of this compartment and further assessed how the altered imprints of various SIMs regulate them prior to therapy. Characterizing these immune profiles in the peripheral blood of cirrhosis patients that received various combinations of IFN-free therapies, we here provide novel findings relevant to the understanding of the HCC immune surveillance process by T cells and how this machinery could be impacted by therapy-induced clearance of HCV. 


\section{Liver Cancer}

It is known that strong and broad anti-tumor T cell responses early in disease are associated with a better prognosis and patient survival $[12,16]$. Our analysis of T cell responses revealed an interesting observation of strong and multispecific GPC-3-specific T cell responses readily detectable in several patients with liver cirrhosis but no ultrasound-detectable cancer,

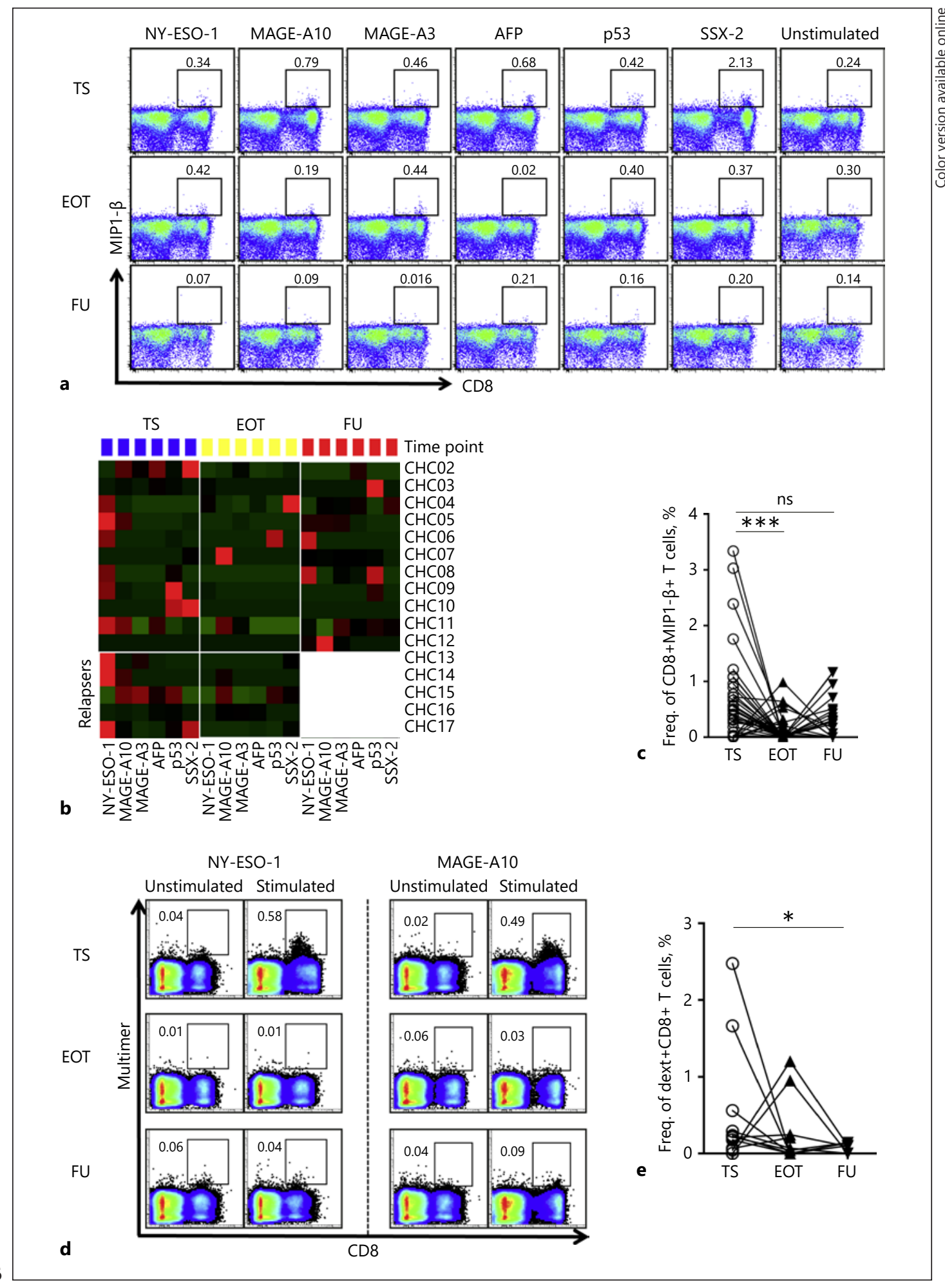

(For legend see next page.) 


\section{Liver

while being less frequent in non-cirrhotic controls. The breadth of $\mathrm{T}$ cell responses that have been described as an independent factor for patient survival $[12,23]$ was also enhanced in liver cirrhosis. Furthermore, MIP1- $\beta$ secretions to stimulations with specific HLA-A2-restricted epitopes of the HCC-associated antigens AFP, NY-ESO-1, MAGE-A3, MAGE-A10, SSX-2, and p53 could be observed in a majority of cirrhosis patients at this time point despite the fact that corresponding expansion of dextramer-specific CD8+ T cells was relatively uncommon. In our cirrhotic cohort without any detectable tumors, it could well be imagined that the multifunctional phenotype of HCC-specific T cells present prior to antiviral therapy are those primed against preneoplastic cells in a way to prevent development or at least delay the progression of these microscopic satellite HCC that missed detection by ultrasound. These findings are not just in conformity with various previous observations [24-28], but have considerable lines of evidence highlighting their biological significance. Recently, anti-GPC-3 chimeric antigen receptor-T cell therapy [29] and the GPC-3-targeted bispecific T cell engager [30] have been shown to be promising candidates for HCC treatment due to their abilities to effectively suppress growth of tumor cells in vitro and in vivo. The antigenic epitopes AFP, NY-ESO-1, MAGE-A3, MAGE-A10, SSX-2, and p53 have also been previously described as highly immunogenic and promising immunotherapeutic target antigens [31]. It is thus conceivable that detectable responses to these immunogenic targets as observed, would contribute to putting cirrhosis patients in a better state of protection against cancer cell proliferation that may crop up during therapy-induced clearance of HCV.

There have been recent debates about a purported mechanism of HCC emergence during DAA therapy that implicates the loss of the prevailing immune cancer surveillance system, particularly by T cells $[32,33]$. Our data do not present striking evidence that this is the case. First, the robust baseline HCC-specific CD4+ T cell response of the cirrhosis patients with no detectable tumors (cirrh-to-No HCC) stayed stable without experiencing any significant disruption upon therapy. Second, although the HCC-specific CD8+ T cell responses significantly tailed off upon therapy, they were not completely jeopardized as they could still be detected at EOT and/or FU at significantly higher magnitudes compared to the increased responses observed in individual cirrh-to-HCC patients at those time points. Third, our data do not support the likelihood that this decline is enough to invariably contribute to clinically evident cases of HCC emergence, especially in a patient cohort that possesses functionally competent T-cell establishment prior to antiviral therapy. For now, it appears that a compromised baseline anti-HCC T-cell response is what presents a significant risk to HCC emergence following IFN-free therapy rather than any direct drug-related effects. Clearly, this warrants

Fig. 6. Fate of TAA epitope-specific CD8+ T cell responses in cirrhosis patients following IFN-free antiviral therapy for hepatitis C. PBMC from cirrhosis patients longitudinally isolated at therapy start (TS), end of therapy (EOT), and follow-up (FU) were stimulated with individual HLA-A2-restricted peptides of AFP137145, NY-ESO-1157-165, MAGE-A3112-120, MAGE-A10254-262, SSX-241-49, and p53149-157. a Exemplary FACS plots from 1 patient showing MIP1- $\beta$ secretion as assessed by intracellular cytokine staining upon stimulation by the six different epitopes. The displayed numbers are indicative of the frequencies of MIP1- $\beta$ producing CD8+ T cells. b Heat map comparing MIP1- $\beta$ secretion by TAA-specific CD8+ T cells analyzed for all epitopes in all patients at TS (blue), EOT (yellow) and FU (red). Patients CHC13-CHC17 as duly marked indicate relapsers. FU values of all responses from the relapsers were excluded in subsequent comparative analysis. c Summary data comparing MIP1- $\beta$ secretions by TAA-specific CD8+ T cells between the three time points. $\mathbf{d}$ Representative FACS stainings of detectable MHC-I multimer-positive TAA-specific CD8+ T cells for two epitopes from 1 patient. e Pooled data comparing the expansion of multimer-positive HCC-specific CD8+ T cells at TS, EOT, and FU. Corresponding background responses were deducted from all stimulated conditions. The Wilcoxon matched-pairs signed-rank test was used for statistical comparisons. CHC, chronic hepatitis C; dext, dextramer. 
further investigations to determine whether the reduction in the HCC immune T cell surveillance system is the cause or the consequence of a therapy-induced viral clearance. The biological importance thereof could further be addressed in a long-term FU study.

Following our observation of the apparent reduction of the prevailing TAA-specific $\mathrm{T}$ cell immune surveillance system in cirrhosis patients at EOT and/or FU upon IFN-free therapy, however, we aimed to interrogate the inflammatory microenvironment for mechanistic cues. Essentially, a balance between the chronic inflammation associated with HCC and immune cell phenotype is what governs the pathogenesis of the malignancy. In this light, the association of serum levels of SIM with the development of HCC was recently demonstrated in DAA-

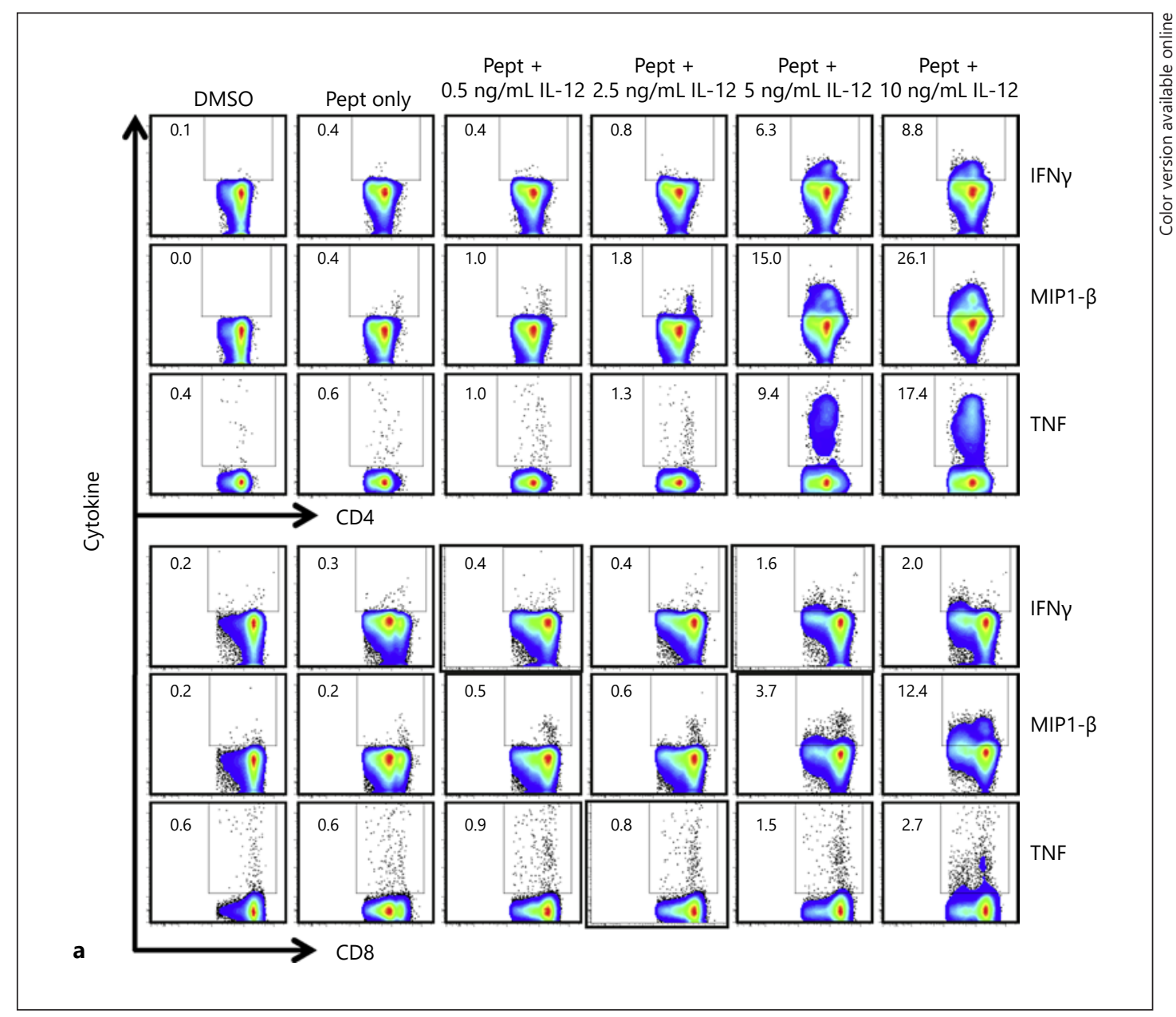

Fig. 7. Effect of SIM stimulation in vitro on TAA-specific T cell responses in cirrhosis patients. PBMC isolated from cirrhosis patients $(n=8)$ before antiviral therapy were stimulated with decreasing concentrations $(10$, $5,2.5$, and $0.5 \mathrm{ng} / \mathrm{mL}$ ) of IL-12 in the presence of GPC-3 overlapping peptides. a Exemplary FACS plots of 1 patient demonstrating cytokine production by TAA-specific CD4+ and CD8+ T cells at different concentrations of IL-12 as indicated. Pooled data illustrating the secretions of IFN $\gamma$ (b), MIP1- $\beta$ (c), and TNF (d) by TAA-specific CD4+ and CD8+ T cells upon IL-12 stimulation in vitro. e Representative FACS plots from 2 patients depicting the pattern of proliferation of TAA-specific CD4+ and CD8+ T cells with reducing concentrations of IL-12 stimulation. $\mathbf{f}$ Summary data of the proliferative responses. $\mathbf{g}$ Differential plasma concentrations of IL-12 in cirrh-to-No HCC and cirrh-to-HCC patients compared between samples collected at TS, EOT, and FU. Statistical tests were performed using Friedman test and Dunn's multiple comparison. For g, the repeated measures one-way ANOVA with Geisser-Greenhouse correction was used. IL-12, interleukin-12; pept, peptide.

(Figure continued on next pages.) 

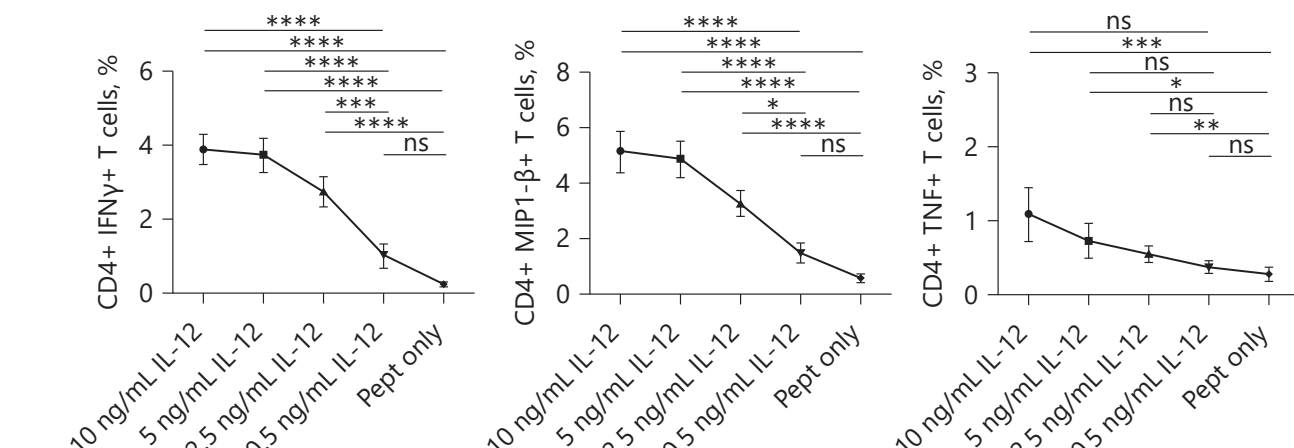

$\lambda^{2} \lambda^{2} \lambda^{2} \lambda^{2}$ तो $b e e^{e^{x}} e^{e^{x^{x}}} e^{e^{x^{x}}} e^{e^{x^{x}}}$
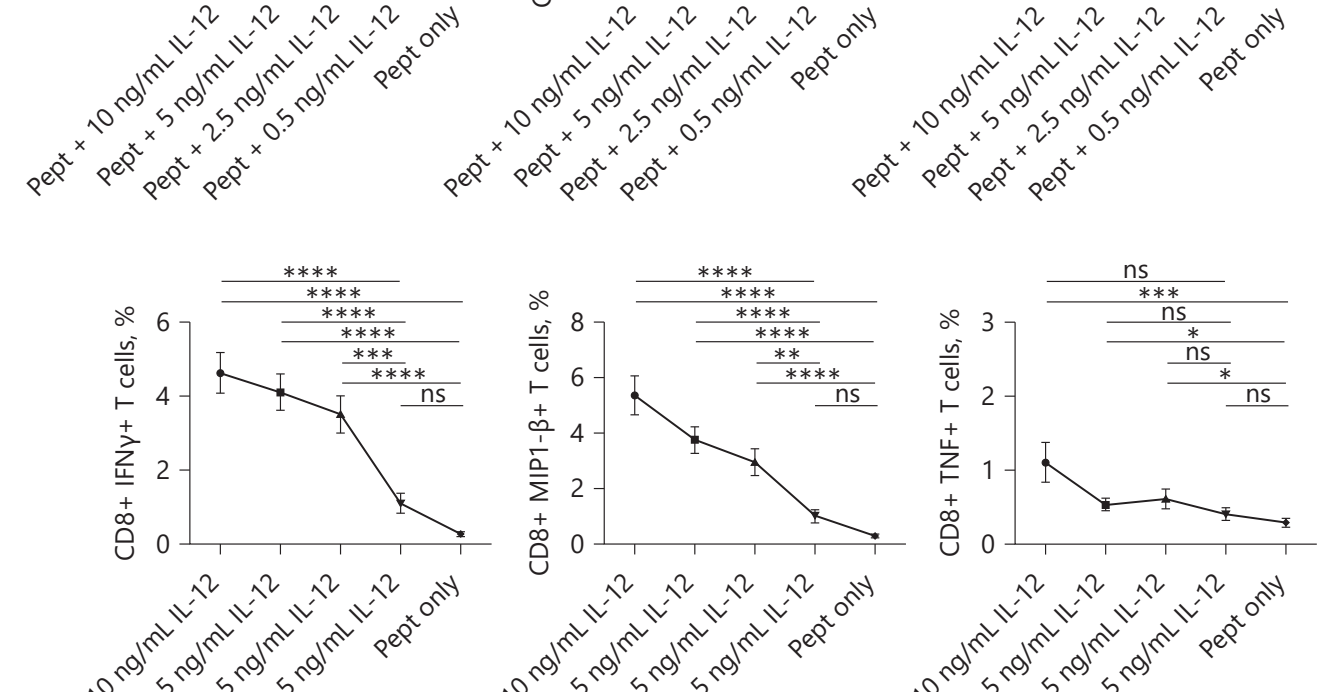



b
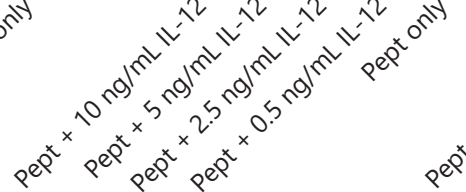

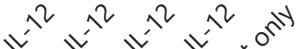

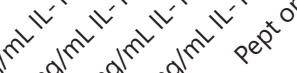



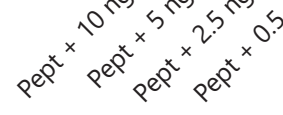

d

Pept $+\quad$ Pept $+\quad$ Pept $+\quad$ Pept +






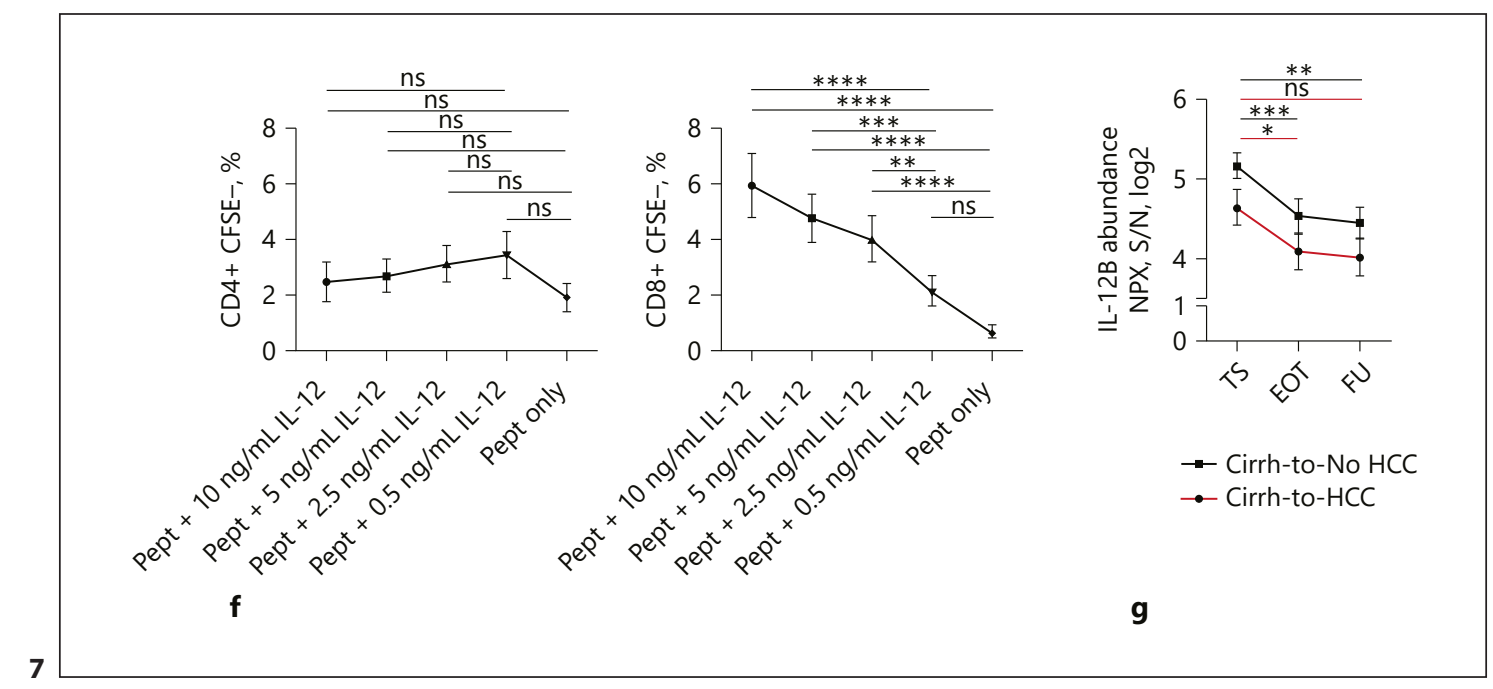

treated patients with HCV infection [34]. It is therefore conceivable that an active treatment that causes significant alterations in inflammatory mediator concentrations in the blood is likely to skew the baseline anti-tumor immune status in favor of tumor progression or its prevention. One of such SIMs which is a classical signal 3 cytokine with receptors expressed on $\mathrm{T}$ cells to enable them respond to shifts in their concentrations in the blood is IL-12. This cytokine, which has monocytes/macrophages, B cells, and dendritic cells as its established sources [35, 36], is also critical for skewing CD4 T cell responses towards a T helper 1 (i.e., IFN $\gamma^{\text {hi }}$, IL-4 ${ }^{\text {low }}$ ) cytokine profile [37]. IL-12 secretion, together with many other SIMs, is known to be triggered and sustained during the immune activation that ensues in both the periphery and hepatic tissues during CHC. Interestingly, IL-12 was not only among the most differentially upregulated SIMs in the plasma of cirrhosis patients relative to the healthy population (Fig. 1b, c), but also the only SIM that normalized among 49 others analyzed in our previous study [18]. Further, IL-12 plasma concentrations in the cirrhosis patients reduced significantly upon therapy-induced HCV clearance. Using this SIM for in vitro stimulation of PBMC from cirrhosis patients, we could show that high IL-12 concentrations augmented the function of HCC-specific T cells, and a lowering of their concentration resulted in a concentrationdependent reduction of functionality. Interestingly, the lack of an in vitro effect of IL-12 as exhibited by the proliferation of HCC-specific CD4+ T cells exactly mirrored the fate of these cells in our longitudinal assessment. These data particularly reveal the profound effect changes in SIM concentrations may generally have on the function of specific immunesurveilling T cells. It further suggests a special importance of IL-12 in regulating anti-tumor immunity in the context of antiviral therapies for hepatitis $\mathrm{C}$.

Inasmuch as this report present several novel findings relevant to the understanding of how HCC progression may be influenced by current IFN-free therapies for hepatitis C, it has considerable limitations that have to be highlighted. First, as this study included only patients who had received IFN-free DAA therapies, it does not address the question of whether there exists a difference between IFN-based treatments and DAA treatments with regard to antitumor immunity in patients with an SVR. Second, this study was based on analysis of only plasma proteins and peripheral blood leukocytes. Even though previous studies indicated a fairly correlative relationship between $\mathrm{T}$ cell response data obtained from blood and those from the liver [12], this current report does not address the possibility of reproducing the findings with intrahepatic samples. Further studies of $\mathrm{T}$ cells and inflammatory responses in 
the liver (e.g., activation of myeloid cells, antigen-presentation, ILC populations, MAIT cells) are thus worth conducting to validate our findings here. Third, this study focused on only GPC-3-specific T cell responses and thus may not reflect the entirety of the immune surveillance machinery of HCC. Although we reckon that GPC-3 is the best TAA for studies like this one because of its high immunogenicity [12] and for it being the center of attention for the recent advances in HCC immunotherapy and adoptive T-cell therapy [30, 29, 38-41], investigation of other TAAs should be considered in future studies.

In summary, our study here demonstrated that HCC-specific T cell responses are readily detectable in patients with liver cirrhosis relative to non-cirrhotic controls. These responses were rather weak in cirrhotic patients who further developed HCC upon DAA therapy, accentuating their important role in inhibiting tumor growth. IFN-free DAA therapy for HCV was associated with a weakening of the strength of HCC-specific CD8+ but not CD4+ T cell responses in cirrhotic patients in vitro. We further provided evidence that the serum inflammatory milieu, which is altered in patients with cirrhosis, may contribute to this compromised response. These data provide a primary base in efforts that would help us accurately define baseline risks of HCC emergence in patients that are to receive antiviral therapies for hepatitis $\mathrm{C}$ and inform a better clinical management practice.

\section{Disclosure Statement}

B.S. has received travel grants from AbbVie, MSD, Shionogi and Gilead.

K.D. has received lecture fees from Gilead, AbbVie, and Merck.

T.C.W. has received lecture fees from Novartis, Roche, and Ipsen.

A.V. has received fees for consultancy and sponsored lectures from Bayer, Lilly, Roche, Novartis, and Amgen.

M.P.M. has received grants and personal fees from AbbVie, Biotest, Boehringer Ingelheim, BristolMyers Squibb, Gilead, GlaxoSmithKline, Janssen, Merck (MSD), Novartis, and Roche and personal fees from Achillion.

M.C. has received lecture fees from AbbVie, Bristol-Myers Squibb, Boehringer Ingelheim Pharma, Gilead, Janssen-Cilag, MSD Sharp \& Dohme/Merck, Roche Diagnostic, Roche Pharma, and Siemens; advisory board fees from AbbVie, Bristol-Myers Squibb, Boehringer Ingelheim Pharma, Gilead, Roche Diagnostic, and Roche Pharma; and data safety board fees from Janssen-Cilag.

H.W. has received grants from AbbVie, Gilead, Roche, Roche Diagnostics, Abbott, Myr, and Eiger and consulting fees or honoraria from AbbVie, Abbott, BMS, Boehringer Ingelheim, Eiger, Gilead, Janssen, Novartis, MSD/Merck, Roche, Roche Diagnostics, and Transgene. In addition, H.W. has received money for board memberships from AbbVie, Abbott, BMS, Boehringer, Eiger, Gilead, Myr, Novartis, and Roche; honoraria for consultancy or speaking from Eiger, Janssen, Siemens, Abbott, AbbVie, Biolex, BMS, Boehringer Ingelheim, ITS, JJ/Janssen-Cilag, Medgenics, Merck/Schering-Plough, Novartis, Roche, Roche Diagnostics, Siemens, Transgene, ViiV; and for lectures, including service on speakers bureaus, from the Falk Foundation and OmnisMed. All other authors report no potential conflicts.

\section{Author Contributions}

S.O.S.: Study concept and design; acquisition of data; analysis and interpretation of data; statistical analysis; drafting of the manuscript; critical revision of the manuscript for important intellectual content; final approval of manuscript.

B.S.: Acquisition of data; critical revision of the manuscript for important intellectual content; final approval of manuscript.

F.M.: Acquisition of data; critical revision of the manuscript for important intellectual content; final approval of manuscript.

M.K.: Acquisition of data; critical revision of the manuscript for important intellectual content; final approval of manuscript. 
K.D.: Acquisition of data; final approval of manuscript.

T.C.W.: Study concept and design; critical revision of the manuscript for important intellectual content; final approval of manuscript.

A.V.: Study concept and design; final approval of manuscript.

M.P.M: Final approval of manuscript.

C.S.F.: Acquisition of data; critical revision of the manuscript for important intellectual content.

M.C.: Interpretation of data; critical revision of the manuscript for important intellectual content; final approval of manuscript.

H.W.: Principal investigator and study supervisor; study concept and design; analysis and interpretation of data; drafting of the manuscript; critical revision of the manuscript for important intellectual content; final approval of manuscript.

\section{References}

1 GBD 2013 Mortality and Causes of Death Collaborators: Global, regional, and national age-sex specific allcause and cause-specific mortality for 240 causes of death, 1990-2013: a systematic analysis for the Global Burden of Disease Study 2013. Lancet 2015;385:117-171.

2 Shlomai A, de Jong YP, Rice CM: Virus associated malignancies: the role of viral hepatitis in hepatocellular carcinoma. Semin Cancer Biol 2014;26:78-88.

3 Razavi H, Waked I, Sarrazin C, et al: The present and future disease burden of hepatitis C virus (HCV) infection with today's treatment paradigm. J Viral Hepat 2014;21(suppl 1):34-59.

4 Backus LI, Belperio PS, Shahoumian TA, Mole LA: Impact of sustained virologic response with direct-acting antiviral treatment on mortality in patients with advanced liver disease. Hepatology DOI: 10.1002/hep.29408.

5 Calleja JL, Crespo J, Rincon D, et al: Effectiveness, safety and clinical outcomes of direct-acting antiviral therapy in HCV genotype 1 infection: results from a Spanish real-world cohort. J Hepatol 2017;66:1138-1148.

6 Ioannou GN, Green PK, Berry K: HCV eradication induced by direct-acting antiviral agents reduces the risk of hepatocellular carcinoma. J Hepatol DOI: 10.1016/j.jhep.2017.08.030.

7 Kanwal F, Kramer J, Asch SM, Chayanupatkul M, Cao Y, El-Serag HB: Risk of hepatocellular cancer in HCV patients treated with direct-acting antiviral agents. Gastroenterology 2017;153:996, 1005.e1.

8 Langhans B, Nischalke HD, Kramer B, et al: Increased peripheral CD4+ regulatory T cells persist after successful direct-acting antiviral treatment of chronic hepatitis C. J Hepatol 2017;66:888-896.

9 Ogawa E, Furusyo N, Nomura H, et al: Short-term risk of hepatocellular carcinoma after hepatitis C virus eradication following direct-acting anti-viral treatment. Aliment Pharmacol Ther 2018;47:104-113.

10 Mettke F, Schlevogt B, Deterding K, et al: Interferon-free therapy of chronic hepatitis C with direct-acting antivirals does not change the short-term risk for de novo hepatocellular carcinoma in patients with liver cirrhosis. Aliment Pharmacol Ther 2018;47:516-525.

11 Reig M, Boix L, Marino Z, Torres F, Forns X, Bruix J: Liver cancer emergence associated with antiviral treatment: an immune surveillance failure? Semin Liver Dis 2017;37:109-118.

12 Flecken T, Schmidt N, Hild S, et al: Immunodominance and functional alterations of tumor-associated antigenspecific CD8+ T-cell responses in hepatocellular carcinoma. Hepatology 2014;59:1415-1426.

13 Mizukoshi E, Nakamoto Y, Tsuji H, Yamashita T, Kaneko S: Identification of alpha-fetoprotein-derived peptides recognized by cytotoxic T lymphocytes in HLA-A24+ patients with hepatocellular carcinoma. Int J Cancer 2006;118:1194-1204.

14 Postow MA, Callahan MK, Wolchok JD: Immune checkpoint blockade in cancer therapy. J Clin Oncol 2015;33: 1974-1982.

15 El-Khoueiry AB, Sangro B, Yau T, et al: Nivolumab in patients with advanced hepatocellular carcinoma (CheckMate 040): an open-label, non-comparative, phase $1 / 2$ dose escalation and expansion trial. Lancet 2017; 389:2492-2502.

16 Galon J, Mlecnik B, Bindea G, et al: Towards the introduction of the "Immunoscore" in the classification of malignant tumours. J Pathol 2014;232:199-209.

17 Schreiber RD, Old LJ, Smyth MJ: Cancer immunoediting: integrating immunity's roles in cancer suppression and promotion. Science 2011;331:1565-1570.

18 Hengst J, Falk CS, Schlaphoff V, et al: Direct-acting antiviral-induced hepatitis C virus clearance does not completely restore the altered cytokine and chemokine milieu in patients with chronic hepatitis C. J Infect Dis 2016;214:1965-1974.

19 Meissner EG, Wu D, Osinusi A, et al: Endogenous intrahepatic IFNs and association with IFN-free HCV treatment outcome. J Clin Invest 2014;124:3352-3363.

20 Suneetha PV, Schlaphoff V, Wang C, et al: Effect of peptide pools on effector functions of antigen-specific CD8+ T cells. J Immunol Methods 2009;342:33-48. 
21 Gisa A, Suneetha PV, Behrendt P, et al: Cross-genotype-specific T-cell responses in acute hepatitis E virus (HEV) infection. J Viral Hepat 2016;23:305-315.

22 Owusu Sekyere S, Suneetha PV, Hardtke S, et al: Type I interferon elevates co-regulatory receptor expression on CMV- and EBV-specific CD8 T cells in chronic hepatitis C. Front Immunol 2015;6:270.

23 Yang J, Murphy TL, Ouyang W, Murphy KM: Induction of interferon-gamma production in Th1 CD4+ T cells: evidence for two distinct pathways for promoter activation. Eur J Immunol 1999;29:548-555.

24 Behboudi S, Alisa A, Boswell S, Anastassiou J, Pathan AA, Williams R: Expansion of anti-AFP Th1 and Tc1 responses in hepatocellular carcinoma occur in different stages of disease. Br J Cancer 2010;102:748-753.

25 Evdokimova VN, Liu Y, Potter DM, Butterfield LH: AFP-specific CD4+ helper T-cell responses in healthy donors and HCC patients. J Immunother 2007;30:425-437.

26 Gehring AJ, Ho ZZ, Tan AT, et al: Profile of tumor antigen-specific CD8 T cells in patients with hepatitis B virusrelated hepatocellular carcinoma. Gastroenterology 2009;137:682-690.

27 Mizukoshi E, Yamashita T, Arai K, et al: Enhancement of tumor-associated antigen-specific T cell responses by radiofrequency ablation of hepatocellular carcinoma. Hepatology 2013;57:1448-1457.

28 Thimme R, Neagu M, Boettler T, et al: Comprehensive analysis of the alpha-fetoprotein-specific CD8+ T cell responses in patients with hepatocellular carcinoma. Hepatology 2008;48:1821-1833.

29 Jiang Z, Jiang X, Chen S, et al: Anti-GPC3-CAR T cells suppress the growth of tumor cells in patient-derived xenografts of hepatocellular carcinoma. Front Immunol 2017;7:690.

30 Bi Y, Jiang H, Wang P, et al: Treatment of hepatocellular carcinoma with a GPC3-targeted bispecific T cell engager. Oncotarget 2017;8:52866-52876.

31 Li KK, Adams DH: Antitumor CD8+ T cells in hepatocellular carcinoma: present but exhausted. Hepatology 2014;59:1232-1234.

32 Llovet JM, Villanueva A: Liver cancer: effect of HCV clearance with direct-acting antiviral agents on HCC. Nat Rev Gastroenterol Hepatol 2016;13:561-562.

33 Reig M, Marino Z, Perello C, et al: Unexpected high rate of early tumor recurrence in patients with HCV-related HCC undergoing interferon-free therapy. J Hepatol 2016;65:719-726.

34 Debes JD, van Tilborg M, Groothuismink ZMA, et al: Levels of cytokines in serum associate with development of hepatocellular carcinoma in patients with HCV infection treated with direct-acting antivirals. Gastroenterology 2018;154:515-517.e3.

35 Liu J, Cao S, Kim S, et al: Interleukin-12: an update on its immunological activities, signaling and regulation of gene expression. Curr Immunol Rev 2005;1:119-137.

36 Trinchieri G: Interleukin-12: a cytokine produced by antigen-presenting cells with immunoregulatory functions in the generation of T-helper cells type 1 and cytotoxic lymphocytes. Blood 1994;84:4008-4027.

37 Heufler C, Koch F, Stanzl U, et al: Interleukin-12 is produced by dendritic cells and mediates T helper 1 development as well as interferon-gamma production by T helper 1 cells. Eur J Immunol 1996;26:659-668.

38 Dargel C, Bassani-Sternberg M, Hasreiter J, et al: T cells engineered to express a T-cell receptor specific for glypican-3 to recognize and kill hepatoma cells in vitro and in mice. Gastroenterology 2015;149:1042-1052.

39 Gao H, Li K, Tu H, et al: Development of T cells redirected to glypican-3 for the treatment of hepatocellular carcinoma. Clin Cancer Res 2014;20:6418-6428.

40 Sun CK, Chua MS, He J, So SK: Suppression of glypican 3 inhibits growth of hepatocellular carcinoma cells through up-regulation of TGF-beta2. Neoplasia 2011;13:735-747.

41 Zhou F, Shang W, Yu X, Tian J: Glypican-3: a promising biomarker for hepatocellular carcinoma diagnosis and treatment. Med Res Rev 2018;38:741-767. 